\title{
TU/e emonownen

\section{Stability analysis of networked control systems using a switched linear systems approach}

\section{Citation for published version (APA):}

Donkers, M. C. F., Heemels, W. P. M. H., Wouw, van de, N., \& Hetel, L. L. (2011). Stability analysis of networked control systems using a switched linear systems approach. IEEE Transactions on Automatic Control, 56(9), 2101-2115. https://doi.org/10.1109/TAC.2011.2107631

DOI:

10.1109/TAC.2011.2107631

Document status and date:

Published: 01/01/2011

\section{Document Version:}

Accepted manuscript including changes made at the peer-review stage

\section{Please check the document version of this publication:}

- A submitted manuscript is the version of the article upon submission and before peer-review. There can be important differences between the submitted version and the official published version of record. People interested in the research are advised to contact the author for the final version of the publication, or visit the $\mathrm{DOI}$ to the publisher's website.

- The final author version and the galley proof are versions of the publication after peer review.

- The final published version features the final layout of the paper including the volume, issue and page numbers.

Link to publication

\section{General rights}

Copyright and moral rights for the publications made accessible in the public portal are retained by the authors and/or other copyright owners and it is a condition of accessing publications that users recognise and abide by the legal requirements associated with these rights.

- Users may download and print one copy of any publication from the public portal for the purpose of private study or research.

- You may not further distribute the material or use it for any profit-making activity or commercial gain

- You may freely distribute the URL identifying the publication in the public portal.

If the publication is distributed under the terms of Article 25fa of the Dutch Copyright Act, indicated by the "Taverne" license above, please follow below link for the End User Agreement:

www.tue.nl/taverne

Take down policy

If you believe that this document breaches copyright please contact us at:

openaccess@tue.nl

providing details and we will investigate your claim. 


\title{
Stability Analysis of Networked Control Systems Using a Switched Linear Systems Approach
}

\author{
M.C.F. Donkers, Student Member, IEEE, W.P.M.H. Heemels Senior Member, IEEE, \\ N. van de Wouw Member, IEEE, and L. Hetel
}

\begin{abstract}
In this paper, we study the stability of Networked Control Systems (NCSs) that are subject to time-varying transmission intervals, time-varying transmission delays and communication constraints. Communication constraints impose that, per transmission, only one node can access the network and send its information. The order in which nodes send their information is orchestrated by a network protocol, such as, the Round-Robin (RR) and the Try-Once-Discard (TOD) protocol. In this paper, we generalise the mentioned protocols to novel classes of socalled 'periodic' and 'quadratic' protocols. By focussing on linear plants and controllers, we present a modelling framework for NCSs based on discrete-time switched linear uncertain systems. This framework allows the controller to be given in discrete time as well as in continuous time. To analyse stability of such systems for a range of possible transmission intervals and delays, with a possible nonzero lower bound, we propose a new procedure to obtain a convex overapproximation in the form of a polytopic system with norm-bounded additive uncertainty. We show that this approximation can be made arbitrarily tight in an appropriate sense. Based on this overapproximation, we derive stability results in terms of Linear Matrix Inequalities (LMIs). We illustrate our stability analysis on the benchmark example of a batch reactor and show how this leads to tradeoffs between different protocols, allowable ranges of transmission intervals and delays. In addition, we show that the exploitation of the linearity of the system and controller leads to a significant reduction in conservatism with respect to existing approaches in the literature.
\end{abstract}

Index Terms-Communication Constraints, Networked Control Systems, Switched Systems, Stability, Time-Varying Systems, Uncertain Systems

\section{INTRODUCTION}

$\mathbf{N}$ ETWORKED Control Systems (NCSs) are systems in which control loops are closed over a real-time communication network. The fact that controllers, sensors, and actuators are not connected through point-to-point connections, but through a multipurpose network offers advantages, such as increased system flexibility, ease of installation and maintenance, and decreased wiring and cost. However, networking

This work is supported by the Dutch Science Foundation (STW) and the Dutch Organisation for Scientific Research (NWO) under the VICI grant "Wireless controls systems: A new frontier in automation", by the European Community through the FP7-ICT-2007-2 thematic programme under the WIDE-224168 project, and by the Embedded Systems Institute, Eindhoven, the Netherlands.

Tijs Donkers, Maurice Heemels, and Nathan van de Wouw are with the Mechanical Engineering department of Eindhoven University of Technoloy, the Netherlands, \{m.c.f.donkers, m.heemels, n.v.d.wouw\} atue.nl.

Laurentiu Hetel is with the Laboratoire d'Automatique, Génie Informatique et Signal (LAGIS) in Lille, France, laurentiu.heteldec-lille.fr.
TABLE I: References that study two networked induced imperfections simultaneously.

\begin{tabular}{c||cc} 
\& & (iv) & (v) \\
\hline \hline (i) & {$[31]$} & \\
(ii) & {$[32],[33]$} & \\
(iii) & {$[34],[35]$} & {$[36]-[40]$}
\end{tabular}

the control system also introduces new challenges, caused by the packet-based data exchange between different parts of the network. Therefore, control algorithms are needed that can handle the communication imperfections and constraints caused by the packet-based communication. The control community is widely aware of this fact, as is evidenced by the broad attention NCSs have received recently, see, e.g., the overview papers [1]-[4].

In general, network-induced communication imperfections and constraints can be categorised into five types:

(i) Quantisation errors in the transmitted signals, due to the finite word length of the transmitted packets.

(ii) Packet dropouts, due to unreliable transmissions.

(iii) Variable sampling/transmission intervals.

(iv) Variable transmission delays.

(v) Communication constraints, i.e., not all sensor and actuator signals can be transmitted at the same time.

It is generally known that any of these phenomena can degrade closed-loop performance or, even worse, can harm closed-loop stability of the control system. It is therefore important to know how these effects influence the stability properties.

Systematic approaches to analyse stability of NCSs subject to only one of these network-induced imperfections are well developed. For instance, the effects of quantisation are studied in [5]-[9], of packet dropouts in [10]-[12], of time-varying transmission intervals and delays in [13]-[17], and [18]-[24], respectively, and of communication constraints in [25]-[28]. However, since in NCSs typically all the aforementioned limitations and constraints are present simultaneously, it is relevant to study the consequences of all these phenomena in a common framework. Unfortunately, fewer results are available that study combinations of these imperfections. References that simultaneously consider two types of network-induced imperfections are given in Table I. Furthermore, [29] considers imperfections of type (i), (iii), (v) and [30] studies type (ii), (iii) and (iv) simultaneously. In this paper, we will focus on the stability of NCSs with time-varying transmission intervals and delays and the presence of communication constraints, 
i.e., type (iii), (iv) and (v) phenomena.

Stability of NCSs subject to communication constraints, time-varying transmission intervals and transmission delays has already been considered in [41], [42]. The communication constraints impose that, per transmission, only one node can access the network and send its information and, hence, a protocol is needed to orchestrate when a certain communication node is given access to the network. Given a protocol, such as the Round-Robin (RR) and the Try-Once-Discard (TOD) protocol, the mentioned papers provide criteria for computing the so-called Maximum Allowable Transmission Interval (MATI) and the Maximum Allowable Delay (MAD). Stability is guaranteed as long as the actual transmission intervals and delays are always smaller than the MATI and MAD, respectively. The difference between the work in [41] and [42], is that in the latter a delay compensation scheme is proposed. This delay compensation requires time stamping of the messages and sending future control signals in larger packets, which is not needed in the more basic emulation based approach, as in [41] and the earlier work without transmission delays in [29], [36]-[40], [43]. Furthermore, the results in [42] have the drawback that they are not applicable to the commonly used Round-Robin protocol, while [41] is.

The work presented in [41], [42] both apply to general nonlinear plants and controllers and are based on a continuoustime modelling paradigm related to hybrid systems as in [44]. However, neither [41], nor [42] include the possibility that the controller is formulated in discrete time. The case of discretetime controllers has been considered in [25], where, however, a fixed transmission interval and no delay are assumed. Another feature of [41], [42] is that, in these works, zero lower bounds on the transmission intervals $h_{k}$ and delays $\tau_{k}$ are considered (i.e., $\left.h_{k} \in\left(0, h_{\mathrm{MATI}}\right], \tau_{k} \in\left[0, \tau_{\mathrm{MAD}}\right]\right)$. The ability to handle discrete-time controllers and nonzero lower bounds on the transmission intervals and delays is highly relevant from a practical point of view, because controllers are typically implemented in a digital and, thus, discrete-time form. Furthermore, finite communication bandwidth always introduce nonzero lower bounds on the transmission intervals and transmission delays. This motivates the need for studying these situations as well, preferably in a nonconservative manner. Although the work presented in [41], [42] is very general and can accommodate for many nonlinear NCSs, their results cannot reduce conservatism when a certain structure is present in the NCS, such as linearity of the controller and plant.

In this paper, we focus on linear plants and linear controllers and study the stability of the corresponding NCS in the presence of communication constraints, time-varying transmission intervals and time-varying delays, where the latter two possibly have a nonzero lower bound. Moreover, we allow for both a continuous-time as well as a discrete-time controller, which requires a different modelling paradigm than in [41], [42], and in the work without transmission delays, [36]-[40]. In particular, we provide techniques for assessing stability of the NCS with time-varying transmission intervals $h_{k} \in[\underline{h}, \bar{h}]$ and timevarying transmission delays $\tau_{k} \in[\underline{\tau}, \bar{\tau}]$ for two well-known protocols, namely, the Round-Robin (RR) protocol and the Try-Once-Discard (TOD) protocol, and their generalisations.
These generalisations consist of the classes of 'periodic' and 'quadratic' protocols, which are formally introduced here. In contrast with [41], [42], we apply a discrete-time modelling framework that leads to a switched linear system model with exponential uncertainty. To properly handle this exponential uncertainty, we provide a polytopic overapproximation for this system. This overapproximation is obtained using a novel procedure that combines ideas from gridding [14], [15] and norm bounding [16]-[18]. Unlike other methodologies for obtaining a convex overapproximation, see, e.g., [14]-[20] and the overview paper [45], we provide a proof that the newly proposed procedure can be made arbitrarily tight in an appropriate sense. Using this overapproximated system, we can assess stability using newly developed conditions based on Linear Matrix Inequalities (LMIs). We will show the effectiveness of the presented approach on the benchmark example of a batch reactor as used in [25], [36]-[39], [41], [43], as well. Moreover, we will show that the linearity of plant and controller can indeed be exploited, which leads to a significant reduction of conservatism with respect to the existing approaches.

The remainder of this paper is organised as follows. After introducing the necessary notational conventions, we introduce the model of the NCS in Section II and propose a method to write it as a discrete-time switched linear uncertain system. We also state a precise problem formulation. Subsequently, in Section III, we provide a procedure to overapproximate the NCS model by a polytopic system with norm-bounded uncertainty. In Section IV, we provide conditions for stability of the NCS in terms of LMIs and reflect in Section V on the conservatism this approach introduces. Finally, we illustrate the stability results using a numerical benchmark example in Section VI and draw conclusions in Section VII. The appendix contains the proofs of the more technical lemmas and theorems.

\section{A. Nomenclature}

The following notational conventions will be used. $\operatorname{diag}\left(A_{1}, \ldots, A_{n}\right)$ denotes a block-diagonal matrix with the entries $A_{1}, \ldots, A_{n}$ on the diagonal and $A^{\top} \in \mathbb{R}^{m \times n}$ denotes the transposed of matrix $A \in \mathbb{R}^{n \times m}$. For a vector $x \in \mathbb{R}^{n}$, we denote by $x^{i}$ the $i$-th component and $\|x\|:=\sqrt{x^{\top} x}$ its Euclidean norm. We denote by $\|A\|:=\sqrt{\lambda_{\max }\left(A^{\top} A\right)}$ its the spectral norm, which is the square-root of the maximum eigenvalue of the matrix $A^{\top} A$. For brevity, we sometimes write symmetric matrices of the form $\left[\begin{array}{cc}A & B \\ B^{\top} & C\end{array}\right]$, as $\left[\begin{array}{cc}A & B \\ \star & C\end{array}\right]$. Finally, by $\lim _{s \downarrow t}$ and $\lim _{s \uparrow t}$, we denote the limit as $s$ approaches $t$ from above and below, respectively, and the convex hull and interior of a set $\mathcal{A}$ are denoted by $\operatorname{co} \mathcal{A}$ and $\operatorname{int} \mathcal{A}$, respectively.

\section{NCS Model AND PRoblem StATEMEnT}

In this section, we present the model describing the Networked Control Systems (NCSs), subject to communication constraints, time-varying transmission intervals and delays. Let us consider the linear time-invariant (LTI) continuous-time 


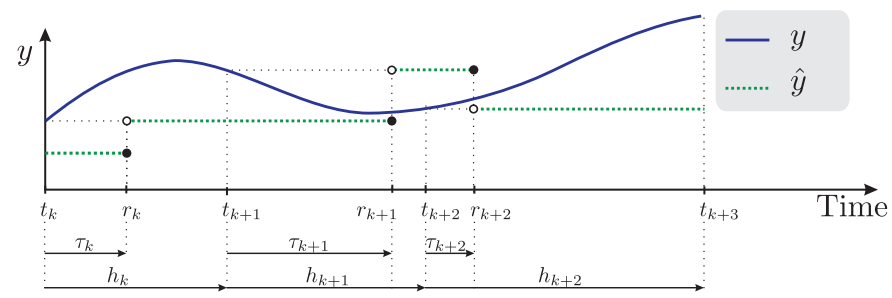

Fig. 1: Illustration of a typical evolution of $y$ and $\hat{y}$.

plant given by

$$
\left\{\begin{aligned}
\frac{\mathrm{d}}{\mathrm{d} t} x^{p}(t) & =A^{p} x^{p}(t)+B^{p} \hat{u}(t) \\
y(t) & =C^{p} x^{p}(t)
\end{aligned}\right.
$$

where $x^{p} \in \mathbb{R}^{n_{p}}$ denotes the state of the plant, $\hat{u} \in \mathbb{R}^{n_{u}}$ the most recently received control variable, $y \in \mathbb{R}^{n_{y}}$ the (measured) output of the plant and $t \in \mathbb{R}^{+}$the time. The controller, also an LTI system, is assumed to be given in either continuous time by

$$
\left\{\begin{aligned}
\frac{\mathrm{d}}{\mathrm{d} t} x^{c}(t) & =A^{c} x^{c}(t)+B^{c} \hat{y}(t) \\
u(t) & =C^{c} x^{c}(t)+D^{c} \hat{y}(t),
\end{aligned}\right.
$$

or in discrete time by

$$
\left\{\begin{array}{l}
x_{k+1}^{c}=A^{c} x_{k}^{c}+B^{c} \hat{y}_{k} \\
u\left(t_{k}\right)=C^{c} x_{k}^{c}+D^{c} \hat{y}\left(t_{k}\right) .
\end{array}\right.
$$

In these descriptions, $x^{c} \in \mathbb{R}^{n_{c}}$ denotes the state of the controller, $\hat{y} \in \mathbb{R}^{n_{y}}$ the most recently received output of the plant and $u \in \mathbb{R}^{n_{u}}$ denotes the controller output. At transmission instant $t_{k}, k \in \mathbb{N}$, (parts of) the outputs of the plant $y\left(t_{k}\right)$ and controller $u\left(t_{k}\right)$ are sampled and are transmitted over the network. We assume that they arrive at instant $r_{k}$, called the arrival instant. The situation described above is illustrated in Fig. 1. In the case we have a discretetime controller (2b), the states of the controller $x_{k+1}^{c}$ are updated using $\hat{y}_{k}:=\lim _{t \downarrow r_{k}} \hat{y}(t)$, i.e., as in [25], directly after $\hat{y}$ is updated. Note that in this case, the update of $x_{k+1}^{c}$ in (2b) has to be performed in the time interval $\left(r_{k}, t_{k+1}\right]$.

Let us now explain in more detail the functioning of the network and define these 'most recently received' $\hat{y}$ and $\hat{u}$ exactly, see also [25], [36]-[41]. The plant is equipped with sensors and actuators that are grouped into $N$ nodes. At each transmission instant $t_{k}, k \in \mathbb{N}$, one node, denoted by $\sigma_{k} \in\{1, \ldots, N\}$, obtains access to the network and transmits its corresponding values. These transmitted values are received and implemented on the controller or the plant at arrival instant $r_{k}$. As in [41], a transmission only occurs after the previous transmission has arrived, i.e., $t_{k+1}>r_{k} \geqslant t_{k}$, for all $k \in \mathbb{N}$. In other words, we consider the sampling interval to be lower bounded and the delays to be smaller than the transmission interval. After each transmission and reception, the values in $\hat{y}$ and $\hat{u}$ are updated with the newly received values, while the other values in $\hat{y}$ and $\hat{u}$ remain the same, as no additional information is received. This leads to the constrained data exchange expressed as

$$
\left\{\begin{array}{l}
\hat{y}(t)=\Gamma_{\sigma_{k}}^{y} y\left(t_{k}\right)+\left(I-\Gamma_{\sigma_{k}}^{y}\right) \hat{y}\left(t_{k}\right) \\
\hat{u}(t)=\Gamma_{\sigma_{k}}^{u} u\left(t_{k}\right)+\left(I-\Gamma_{\sigma_{k}}^{u}\right) \hat{u}\left(t_{k}\right)
\end{array}\right.
$$

for all $t \in\left(r_{k}, r_{k+1}\right]$, where $\Gamma_{\sigma_{k}}:=\operatorname{diag}\left(\Gamma_{\sigma_{k}}^{y}, \Gamma_{\sigma_{k}}^{u}\right)$ is a diagonal matrix, given by

$$
\Gamma_{i}=\operatorname{diag}\left(\gamma_{i, 1}, \ldots, \gamma_{i, n_{y}+n_{u}}\right) .
$$

when $\sigma_{k}=i$. In (4), the elements $\gamma_{i, j}$, with $i \in\{1, \ldots, N\}$ and $j \in\left\{1, \ldots, n_{y}\right\}$, are equal to one, if plant output $y^{j}$ is in node $i$, elements $\gamma_{i, j+n_{y}}$, with $i \in\{1, \ldots, N\}$ and $j \in$ $\left\{1, \ldots, n_{u}\right\}$, are equal to one, if controller output $u^{j}$ is in node $i$, and are zero elsewhere.

The value of $\sigma_{k} \in\{1, \ldots, N\}$ in (3) indicates which node is given access to the network at transmission instant $t_{k}, k \in \mathbb{N}$. Indeed, (3) reflects that the values in $\hat{u}$ and $\hat{y}$ corresponding to node $\sigma_{k}$ are updated just after $r_{k}$, with the corresponding transmitted values at time $t_{k}$, while the others remain the same. A scheduling protocol determines the sequence $\left(\sigma_{0}, \sigma_{1}, \ldots\right)$ and particular protocols will be made explicit later.

The transmission instants $t_{k}$, as well as the arrival instants $r_{k}, k \in \mathbb{N}$ are not necessarily distributed equidistantly in time. Hence, both the transmission intervals $h_{k}:=t_{k+1}-t_{k}$ and the transmission delays $\tau_{k}:=r_{k}-t_{k}$ are varying in time, as is also illustrated in Fig. 1. We assume that the variations in the transmission interval and delays are bounded and are contained in the sets $[\underline{h}, \bar{h}]$ and $[\underline{\tau}, \bar{\tau}]$, respectively, with $\bar{h} \geqslant \underline{h}>0$ and $\bar{\tau} \geqslant \underline{\tau} \geqslant 0$. Since we assumed that each transmission delay $\tau_{k}$ is smaller than the corresponding transmission interval $h_{k}$, we have that $\left(h_{k}, \tau_{k}\right) \in \Theta$, for all $k \in \mathbb{N}$, where

$$
\Theta:=\left\{(h, \tau) \in \mathbb{R}^{2} \mid h \in[\underline{h}, \bar{h}], \tau \in[\underline{\tau}, \min \{h, \bar{\tau}\})\right\} .
$$

Remark II.1 In the above reasoning, we implicitly assumed that packet loss does not occur, similar to, e.g., [25], [37], [39], [40]. However, we could accommodate for packet dropouts by modelling them as prolongations of the transmission interval, as done in [38], [41]. This means that if we assume that there is a bound $\delta \in \mathbb{N}$ on the maximum number of successive dropouts, and we have stability of the NCS for $\left(h_{k}, \tau_{k}\right) \in \Theta$, for all $k \in \mathbb{N}$, in the case without dropouts, then the NCS with dropouts is still guaranteed to be stable for $\left(h_{k}, \tau_{k}\right) \in \Theta^{\prime}$, for all $k \in \mathbb{N}$, where

$$
\Theta^{\prime}:=\left\{(h, \tau) \in \mathbb{R}^{2} \mid h \in\left[\underline{h}, \bar{h}^{\prime}\right], \tau \in[\underline{\tau}, \min \{h, \bar{\tau}\})\right\}
$$

in which $\bar{h}^{\prime}:=\frac{\bar{h}}{\delta+1}$.

\section{A. The NCS as a discrete-time switched uncertain system}

To analyse stability of the NCS described above, we transform it into a discrete-time model. In this framework, we need a discrete-time equivalent of (1) and also of (2a) in case a continuous-time controller is used. To arrive at this description, let us first define the network-induced error as

$$
\left\{\begin{array}{l}
e^{y}(t):=\hat{y}(t)-y(t) \\
e^{u}(t):=\hat{u}(t)-u(t) .
\end{array}\right.
$$

The discrete-time switched uncertain system can now be obtained by describing the evolution of the states between $t_{k}$ and $t_{k+1}=t_{k}+h_{k}$. In order to do so, we define $x_{k}^{p}:=x^{p}\left(t_{k}\right)$, $u_{k}:=u\left(t_{k}\right), \hat{u}_{k}:=\lim _{t \downarrow r_{k}} \hat{u}(t)$ and $e_{k}^{u}:=e^{u}\left(t_{k}\right)$. Since $\hat{u}$, as in (3), is a piecewise constant left-continuous signal, i.e., 


$$
\bar{x}_{k+1}=\underbrace{\left[\begin{array}{cc}
A_{h_{k}}+E_{h_{k}} B D C & E_{h_{k}} B D-E_{h_{k}-\tau_{k}} B \Gamma_{\sigma_{k}} \\
C\left(I-A_{h_{k}}-E_{h_{k}} B D C\right) & I-D^{-1} \Gamma_{\sigma_{k}}+C\left(E_{h_{k}-\tau_{k}} B \Gamma_{\sigma_{k}}-E_{h_{k}} B D\right)
\end{array}\right]}_{=: \tilde{A}_{\sigma_{k}, h_{k}, \tau_{k}}} \bar{x}_{k}
$$

$\lim _{s \uparrow t} \hat{u}(s)=\hat{u}(t)$, we can write $\hat{u}_{k-1}=\lim _{t \downarrow r_{k-1}} \hat{u}(t)=$ $\hat{u}\left(r_{k}\right)=\hat{u}\left(t_{k}\right)$. This allows us to write the exact discretisation of (1) as follows:

$$
\begin{array}{r}
x_{k+1}^{p}=e^{A^{p} h_{k}} x_{k}^{p}+\int_{0}^{h_{k}} e^{A^{p}\left(h_{k}-s\right)} B^{p} \hat{u}\left(t_{k}+s\right) d s \\
=e^{A^{p} h_{k}} x_{k}^{p}+\int_{0}^{\tau_{k}} e^{A^{p}\left(h_{k}-s\right)} d s B^{p} \hat{u}_{k-1} \\
\quad+\int_{\tau_{k}}^{h_{k}} e^{A^{p}\left(h_{k}-s\right)} d s B^{p} \hat{u}_{k} .
\end{array}
$$

As (3) and (7) yield $\hat{u}_{k-1}=u_{k}+e_{k}^{u}$ and $\hat{u}_{k-1}-\hat{u}_{k}=\Gamma_{\sigma_{k}}^{u} e_{k}^{u}$, (8) can be rewritten as

$$
\begin{gathered}
x_{k+1}^{p}=e^{A^{p} h_{k}} x_{k}^{p}+\int_{h_{k}-\tau_{k}}^{h_{k}} e^{A^{p} s} d s B^{p} \hat{u}_{k-1} \\
\quad+\int_{0}^{h_{k}-\tau_{k}} e^{A^{p} s} d s B^{p} \hat{u}_{k} \\
=e^{A^{p} h_{k}} x_{k}^{p}+\int_{0}^{h_{k}} e^{A^{p} s} d s B^{p} \hat{u}_{k-1} \\
+\int_{0}^{h_{k}-\tau_{k}} e^{A^{p} s} d s B^{p}\left(\hat{u}_{k}-\hat{u}_{k-1}\right) \\
=e^{A^{p} h_{k}} x_{k}^{p}+\int_{0}^{h_{k}} e^{A^{p} s} d s B^{p}\left(u_{k}+e_{k}^{u}\right) \\
-\int_{0}^{h_{k}-\tau_{k}} e^{A^{p} s} d s B^{p} \Gamma_{\sigma_{k}}^{u} e_{k}^{u} .
\end{gathered}
$$

A discretised equivalent of (2a) is obtained in a similar fashion by defining $x_{k}^{c}:=x^{c}\left(t_{k}\right), y_{k}:=y\left(t_{k}\right), e_{k}^{y}:=e^{y}\left(t_{k}\right), \hat{y}_{k}:=$ $\lim _{t \downarrow r_{k}} \hat{y}(t)$, and observing $\hat{y}_{k-1}=\hat{y}\left(t_{k}\right)$, and is given by

$$
\begin{aligned}
x_{k+1}^{c}=e^{A^{c} h_{k}} x_{k}^{c}+\int_{0}^{h_{k}} e^{A^{c} s} d s B^{c}\left(y_{k}+e_{k}^{y}\right) \\
-\int_{0}^{h_{k}-\tau_{k}} e^{A^{c} s} d s B^{c} \Gamma_{\sigma_{k}}^{y} e_{k}^{y} .
\end{aligned}
$$

We now present three different models, each describing a particular NCS. The first and the second model cover the situation where both the plant and the controller outputs are transmitted over the network, differing by the fact that the controller is given by $(2 a)$ and $(2 b)$, respectively. In the third model, it is assumed that the controller is given by (2a) and that only the plant outputs $y$ are transmitted over the network and $u$ are sent continuously via an ideal nonnetworked connection. We include this particular case, because it is often used in examples in NCS literature (see, e.g., the benchmark example in [25], [36]-[39], [41]) and it allows us to compare our methodology to the existing ones.
1) The NCS model with controller (2a): For an NCS having controller (2a), the complete NCS model is obtained by combining (3), (7), (9), and (10) and defining

$$
\bar{x}_{k}:=\left[\begin{array}{llll}
x_{k}^{p \top} & x_{k}^{c \top} & e_{k}^{y \top} & e_{k}^{u \top}
\end{array}\right]^{\top} .
$$

This results in the discrete-time model (12), as shown on the top of this page, in which $\tilde{A}_{\sigma_{k}, h_{k}, \tau_{k}} \in \mathbb{R}^{n \times n}$, with $n=n_{p}+$ $n_{c}+n_{y}+n_{u}$, and

$$
\begin{array}{rlrl}
A_{h_{k}} & :=\operatorname{diag}\left(e^{A^{p} h_{k}}, e^{A^{c} h_{k}}\right), \quad B & :=\left[\begin{array}{cc}
0 & B^{p} \\
B^{c} & 0
\end{array}\right], \\
C & :=\operatorname{diag}\left(C^{p}, C^{c}\right), & D & :=\left[\begin{array}{cc}
I & 0 \\
D^{c} & I
\end{array}\right] \\
E_{\rho} & :=\operatorname{diag}\left(\int_{0}^{\rho} e^{A^{p} s} d s, \int_{0}^{\rho} e^{A^{c} s} d s\right), \quad \rho \in \mathbb{R} .
\end{array}
$$

2) The NCS model with controller (2b): For an NCS having controller (2b), the complete NCS model is obtained by combining (2b), (3), (7), and (9), also resulting in (12), in which now

$$
\begin{aligned}
A_{h_{k}} & :=\operatorname{diag}\left(e^{A^{p} h_{k}}, A^{c}\right), & B & :=\left[\begin{array}{cc}
0 & B^{p} \\
B^{c} & 0
\end{array}\right], \\
C & :=\operatorname{diag}\left(C^{p}, C^{c}\right), & D & :=\left[\begin{array}{cc}
I & 0 \\
D^{c} & I
\end{array}\right], \\
E_{\rho} & :=\operatorname{diag}\left(\int_{0}^{\rho} e^{A^{p} s} d s, I\right), & \rho & \in \mathbb{R} .
\end{aligned}
$$

3) The NCS model if only y is transmitted over the network: In this case we assume that only the outputs of the plant are transmitted over the network and the controller communicates its values continuously and without delay. We therefore have that $u(t)=\hat{u}(t)$, for all $t \in \mathbb{R}^{+}$, which allows us to combine (1) and (2a), yielding

$$
\left[\begin{array}{c}
\dot{x}^{p}(t) \\
\dot{x}^{c}(t)
\end{array}\right]=\left[\begin{array}{cc}
A^{p} & B^{p} C^{c} \\
0 & A^{c}
\end{array}\right]\left[\begin{array}{l}
x^{p}(t) \\
x^{c}(t)
\end{array}\right]+\left[\begin{array}{c}
B^{p} D^{c} \\
B^{c}
\end{array}\right] \hat{y}(t) .
$$

Since $\hat{y}$ is still updated according to (3), we can describe the evolution of the states between $t_{k}$ and $t_{k+1}=t_{k}+h_{k}$ in a similar fashion as in (9). In this case, (11) reduces to

$$
\bar{x}_{k}:=\left[\begin{array}{lll}
x_{k}^{p \top} & x_{k}^{c \top} & e_{k}^{y \top}
\end{array}\right]^{\top},
$$

resulting in (12), in which

$$
\begin{array}{rlrl}
A_{h_{k}} & :=e^{\left[\begin{array}{cc}
A^{p} & B^{p} C^{c} \\
0 & A^{c}
\end{array}\right] h_{k}}, & B:=\left[\begin{array}{c}
B^{p} D^{c} \\
B^{c}
\end{array}\right], \\
C & :=\left[\begin{array}{cc}
C^{p} & 0
\end{array}\right], & D & :=I, \\
E_{\rho} & :=\int_{0}^{\rho} e^{\left[\begin{array}{cc}
A^{p} & B^{p} C^{c} \\
0 & A^{c}
\end{array}\right] s} d s, & \rho \in \mathbb{R} .
\end{array}
$$




\section{B. Protocols as a Switching Function}

Based on the previous modelling steps, the NCS is formulated as a discrete-time switched uncertain system (12). In this framework, protocols are considered as the switching function determining $\sigma_{k}$. We consider two commonly used protocols, see [36]-[41], [43], namely the Try-Once-Discard (TOD) and the Round-Robin (RR) protocol and generalise these into two novel classes of protocols, named 'quadratic' and 'periodic' protocols.

1) Quadratic Protocols: A quadratic protocol is a protocol, for which the switching function can be written as

$$
\sigma_{k}=\arg \min _{i=1, \ldots, N} \bar{x}_{k}^{\top} P_{i} \bar{x}_{k}
$$

where $P_{i}, i \in\{1, \ldots, N\}$, are certain given matrices. In case two nodes have the same maximal values, one of them can be chosen arbitrarily. In fact, the well-known TOD protocol, sometimes also called Maximum Error First (MEF) protocol, belongs to this class of protocols. In this protocol, the node that has the largest network-induced error, i.e., the difference between the most recently transmitted values and its current values of the signals corresponding to the node, is granted access to the network. We can arrive at the TOD protocol by adopting the following structure in the $P_{i}$ matrices:

$$
P_{i}=\bar{P}-\operatorname{diag}\left(0, \Gamma_{i}\right)
$$

in which $\Gamma_{i}, i \in\{1, \ldots, N\}$, is given by (4). Furthermore, if we define $\tilde{e}_{k}^{i}:=\Gamma_{i} e_{k}$, where $e_{k}:=\left[e_{k}^{y \top}, e_{k}^{u \top}\right]^{\top},(18)$ becomes

$$
\begin{aligned}
\sigma_{k}=\arg \min \left\{-e_{k}^{\top} \Gamma_{1} e_{k}, \ldots,-e_{k}^{\top} \Gamma_{N} e_{k}\right\} \\
=\arg \max \left\{\left\|\tilde{e}_{k}^{1}\right\|, \ldots,\left\|\tilde{e}_{k}^{N}\right\|\right\},
\end{aligned}
$$

which is the TOD protocol.

2) Periodic Protocols: Another class of protocols that is considered in this paper is the class of so-called periodic protocols. A periodic protocol is a protocol that satisfies for some $\tilde{N} \in \mathbb{N}$

$$
\sigma_{k+\tilde{N}}=\sigma_{k}
$$

for all $k \in \mathbb{N} . \tilde{N}$ is then called the period of the protocol. Actually, the well-known RR protocol belongs to this class and is defined by

$$
\left\{\sigma_{1}, \ldots, \sigma_{N}\right\}=\{1, \ldots, N\},
$$

and period $\tilde{N}=N$, i.e., during each period of the protocol every node has access to the network exactly once.

The above modelling approach now provides a description of the NCS system in the form of a discrete-time switched linear uncertain system given by (12) and one of the protocols, characterised by (18) or (21). The system switches between $N$ linear uncertain systems and the switching is due to the fact that only one node accesses the network at each transmission instant. The uncertainty is caused by the fact that the transmission intervals and the transmission delays $\left(h_{k}, \tau_{k}\right) \in \Theta$ are varying over time.

\section{Stability of the NCS}

The problem studied in this paper is to determine the stability of the continuous-time NCS, given by (1), (2a) or (2b), (3), and (7), with protocols satisfying (18) or (21) given the bounds $[\underline{h}, \bar{h}]$ and $[\underline{\tau}, \bar{\tau}]$, or to find bounds that guarantee stability. Let us now formally define stability for this continuous-time NCS.

Definition II.2 The continuous-time NCS given by (1), (2a) or (2b), (3), and (7), with protocols satisfying (18) or (21), having states $\bar{x}(t):=\left[\begin{array}{llll}x^{p^{\top}}(t) & x^{c \top}(t) & e^{y^{\top}}(t) & e^{u \top}(t)\end{array}\right]^{\top} \in$ $\mathbb{R}^{n}$, is said to be Uniformly Globally Exponentially Stable (UGES) if there exist $\tilde{c} \geqslant 0$ and $\tilde{\lambda}>0$, such that for any initial condition $\bar{x}(0)$, any sequence of transmission intervals $\left(h_{0}, h_{1}, \ldots\right)$, and any sequence of transmission delays $\left(\tau_{0}, \tau_{1}, \ldots\right)$, with $\left(h_{k}, \tau_{k}\right) \in \Theta$, for all $k \in \mathbb{N}$, it holds that

$$
\|\bar{x}(t)\| \leqslant \tilde{c}\|\bar{x}(0)\| e^{-\tilde{\lambda} t}, \quad \forall t \in \mathbb{R}^{+} .
$$

Stability of the continuous-time NCS can be analysed by assessing stability of the discrete-time uncertain switched linear system (12) with switching functions satisfying (18) or (21), as we will show. Let us now formally define stability of this discrete-time system.

Definition II.3 System (12) with switching sequences satisfying (18) or (21) is said to be Uniformly Globally Exponentially Stable (UGES) if there exist $c \geqslant 0$ and $0 \leqslant \lambda<1$, such that for any initial condition $\bar{x}_{0} \in \mathbb{R}^{n}$, any sequence of transmission intervals $\left(h_{0}, h_{1}, \ldots\right)$, and any sequence of transmission delays $\left(\tau_{0}, \tau_{1}, \ldots\right)$, with $\left(h_{k}, \tau_{k}\right) \in \Theta$, for all $k \in \mathbb{N}$, it holds that

$$
\left\|\bar{x}_{k}\right\| \leqslant c\left\|\bar{x}_{0}\right\| \lambda^{k}, \quad \forall k \in \mathbb{N} .
$$

Since the discrete-time switched uncertain linear system (12) with switching sequences satisfying (18) or (21) is formulated in discrete time, we can only assess stability at the transmission instants. However, states of the plant (1) and controller (2a) actually evolve in continuous time. In the next lemma, we state that UGES of the discrete-time NCS model implies UGES of the continuous-time NCS.

Lemma II.4 Assume the discrete-time system (12) with switching sequences satisfying (18) or (21) is UGES, then the continuous-time NCS given by (1), (2a) or (2b), (3), and (7), with protocols satisfying (18) or (21) is also UGES.

Proof: The proof is given in the Appendix.

This lemma states that it suffices to consider the discretetime model (12) with switching sequences satisfying (18) or (21) to assess UGES of the continuous-time NCS system.

\section{OBTAINING A CONVEX OVERAPPROXIMATION}

In the previous section, we obtained an NCS model in the form of a switched uncertain system. However, the form as in (12) is not really convenient to develop efficient techniques for stability analysis due to the nonlinear dependence of $\tilde{A}_{\sigma_{k}, h_{k}, \tau_{k}}$ on the uncertain parameters $h_{k}$ and $\tau_{k}$. Therefore, we will 
provide a procedure that overapproximates system (12) by a polytopic system with a norm-bounded additive uncertainty of the form

$$
\bar{x}_{k+1}=\left(\sum_{l=1}^{L} \alpha_{k}^{l} \bar{A}_{\sigma_{k}, l}+\bar{B} \Delta_{k} \bar{C}_{\sigma_{k}}\right) \bar{x}_{k},
$$

where $\bar{A}_{\sigma, l} \in \mathbb{R}^{n \times n}, \bar{B} \in \mathbb{R}^{n \times m}, \bar{C}_{\sigma} \in \mathbb{R}^{m \times n}$, for $\sigma \in$ $\{1, \ldots, N\}$ and $l \in\{1, \ldots, L\}$, with $L$ the number of vertices of the polytope. Furthermore, $\alpha_{k}=\left[\alpha_{k}^{1} \ldots \alpha_{k}^{L}\right]^{\top} \in \mathcal{A}$, $k \in \mathbb{N}$, denotes an unknown time-varying vector with

$$
\mathcal{A}=\left\{\alpha \in \mathbb{R}^{L} \mid \sum_{l=1}^{L} \alpha^{l}=1, \alpha^{l} \geqslant 0, l \in\{1, \ldots, L\}\right\}
$$

and $\Delta_{k} \in \boldsymbol{\Delta}, k \in \mathbb{N}$, where $\boldsymbol{\Delta}$ is a norm-bounded set of matrices in $\mathbb{R}^{m \times m}$ that describes the additive uncertainty. This additive uncertainty can have some specific structure, as we will see below. The model (25) should be an overapproximation of (12) in the sense that for all $\sigma \in\{1, \ldots, N\}$, it holds that

$$
\begin{aligned}
\left\{\tilde{A}_{\sigma, h, \tau} \mid\right. & (h, \tau) \in \Theta\} \\
& \subseteq\left\{\sum_{l=1}^{L} \alpha^{l} \bar{A}_{\sigma, l}+\bar{B} \Delta \bar{C}_{\sigma} \mid \alpha \in \mathcal{A}, \Delta \in \Delta\right\} .
\end{aligned}
$$

In this paper, we use the gridding idea of [14], [15] to obtain, for a fixed $\sigma, \bar{A}_{\sigma, l}$ by evaluating $\tilde{A}_{\sigma, h, \tau}$ of (12) at a collection of selected pairs of transmission intervals and transmission delays $\left(\tilde{h}_{l}, \tilde{\tau}_{l}\right) \in \Theta, l \in\{1, \ldots, L\}$. Hence, we take $\bar{A}_{\sigma, l}:=$ $\tilde{A}_{\sigma, \tilde{h}_{l}, \tilde{\tau}_{l}}$ in (25), with $l \in\{1, \ldots, L\}$. However, contrary to [14], [15], we choose to allow for convex combinations of the vertices, whereas in [14], [15] the system switches between the vertices only. Moreover, we construct a norm-bounded additive uncertainty $\Delta \in \Delta$ to capture the remaining approximation error, as done in, e.g., [16]-[18]. By comparing $\tilde{A}_{\sigma, h, \tau}$ with the convex combinations of the vertices instead of with the vertices alone, we obtain smaller bounds on the additive uncertainty than in [14]-[16], [18].

By specifying $\left(\tilde{h}_{l}, \tilde{\tau}_{l}\right), l \in\{1, \ldots, L\}$, and thereby determining $\bar{A}_{\sigma, l}$, it only remains to show how to choose $\bar{B} \Delta \bar{C}_{\sigma}$ in (25) and $\Delta$ in order to satisfy (27). This additive uncertainty is used to capture the approximation error between the original system (12) and the polytopic system

$$
\bar{x}_{k+1}=\sum_{l=1}^{L} \alpha_{k}^{l} \bar{A}_{\sigma_{k}, l} \bar{x}_{k} .
$$

In order for (27) to hold, for each triple $(\sigma, h, \tau)$, with $\sigma \in$ $\{1, \ldots, N\}$ and $(h, \tau) \in \Theta$, there should exist some $\alpha \in \mathcal{A}$ and $\Delta \in \boldsymbol{\Delta}$, such that

$$
\tilde{A}_{\sigma, h, \tau}-\sum_{l=1}^{L} \alpha^{l} \bar{A}_{\sigma, l}=\bar{B} \Delta \bar{C}_{\sigma} .
$$

Hence, we should determine the worst-case distance between the real system (12) and the polytopic system (28), leading to an upper bound on the approximation error. To obtain such an upper bound, we partition $\Theta$ into $M$ triangles $\mathcal{S}_{1}, \ldots, \mathcal{S}_{M}$, see Fig. 2, and we compare $\tilde{A}_{\sigma, h, \tau}$, for $(h, \tau) \in \mathcal{S}_{m}$, with

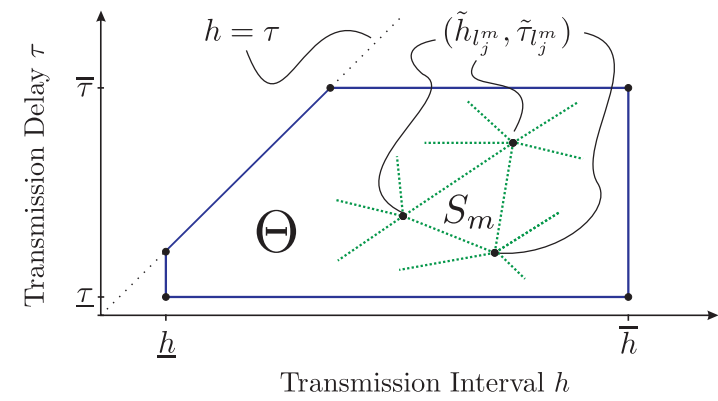

Fig. 2: The partitioning of $\Theta$ into triangles $\mathcal{S}_{m}$.

$\left\{\sum_{j=1}^{3} \tilde{\alpha}^{j} \bar{A}_{\sigma, l_{j}^{m}} \mid \sum_{j=1}^{3} \tilde{\alpha}^{j}=1, \quad \tilde{\alpha}^{j} \geqslant 0, j \in\{1,2,3\}\right\}$, where $\left(\tilde{h}_{l_{j}^{m}}, \tilde{\tau}_{l_{j}^{m}}\right), j=\{1,2,3\}$, denote the vertices (with vertex index $l_{j}^{m} \in\{1, \ldots, L\}, j \in\{1,2,3\}$ and $m \in$ $\{1, \ldots, M\})$ of triangle $\mathcal{S}_{m}$. This allows us to construct the right-hand side of (29) by computing the worst-case distance. Note that it is always possible to partition $\Theta$ into triangles, as $\Theta$ is a convex polytope. We will, however, also provide a systematic procedure to obtain a suitable partitioning.

A specific feature of the overapproximation presented in this paper is that, contrary to [14]-[20], it can be made arbitrarily tight, i.e., besides that (27) holds, it also holds that

$$
\begin{aligned}
& \left\{\sum_{l=1}^{L} \alpha^{l} \bar{A}_{\sigma, l}+\bar{B} \Delta \bar{C}_{\sigma} \mid \alpha \in \mathcal{A}, \Delta \in \Delta\right\} \\
& \quad \subseteq \operatorname{co}\left\{\tilde{A}_{\sigma, h, \tau} \mid(h, \tau) \in \Theta\right\}+\{\bar{\Delta} \mid\|\bar{\Delta}\| \leqslant \varepsilon\},
\end{aligned}
$$

for each $\sigma \in\{1, \ldots, N\}$, in which $\varepsilon>0$ can be chosen arbitrarily small. This can be achieved by increasing the number of pairs $\left(\tilde{h}_{l}, \tilde{\tau}_{l}\right) \in \Theta, l \in\{1, \ldots, L\}$, in a welldistributed fashion. The fact that (30) can be ensured to hold for an arbitrarily small $\varepsilon>0$ is important, as it allows us to show that the existence of a Lyapunov function of a particular type for (12) is equivalent to the existence of a Lyapunov function of the same type for (25). Since we will indeed show that (30) can be guaranteed for any choice of $\varepsilon>0$, we can let the introduced conservatism in the overapproximation vanish. We will formalise this result in Section V.

We now formalise the procedure to obtain a convex overapproximation as outlined above. The procedure results in a tight overapproximation, by adding pairs $\left(\tilde{h}_{l}, \tilde{\tau}_{l}\right) \in \Theta$ until $\varepsilon \leqslant \varepsilon_{u}$ is achieved for an user-specified threshold $\varepsilon_{u}>0$, such that (30) holds with $\varepsilon \leqslant \varepsilon_{u}$.

\section{Procedure III.1}

Step 1 Choose a desired $\varepsilon_{u}>0$. Furthermore, select distinct pairs $\left(\tilde{h}_{l}, \tilde{\tau}_{l}\right) \in \Theta, l \in\{1, \ldots, L\}$, such that $\operatorname{co} \mathcal{G}=\Theta$, where $\mathcal{G}=\cup_{l=1}^{L}\left\{\left(\tilde{h}_{l}, \tilde{\tau}_{l}\right)\right\}$. Now partition $\Theta$ into $M$ triangles $\mathcal{S}_{m}, m \in\{1, \ldots, M\}$, such that, for each $\mathcal{S}_{m} \in \mathcal{H}$, where $\mathcal{H}=\left\{\mathcal{S}_{1}, \ldots, \mathcal{S}_{M}\right\}$, it holds that

$$
\mathcal{S}_{m}=\operatorname{co}\left\{\left(\tilde{h}_{l_{1}^{m}}, \tilde{\tau}_{1}^{m}\right),\left(\tilde{h}_{l_{2}^{m}}, \tilde{\tau}_{l_{2}^{m}}\right),\left(\tilde{h}_{l_{3}^{m}}, \tilde{\tau}_{3}^{m}\right)\right\},
$$

where $l_{j}^{m} \in\{1, \ldots, L\}, j \in\{1,2,3\}$. Hence, $\left(\tilde{h}_{l_{j}^{m}}, \tilde{\tau}_{l_{j}^{m}}\right) \in \mathcal{G}, j \in\{1,2,3\}$ are the vertices of the triangle $\mathcal{S}_{m}$. Moreover, for all $m, p \in\{1, \ldots, M\}$ and $p \neq m$, int $\mathcal{S}_{p} \cap \operatorname{int} \mathcal{S}_{m}=\emptyset, \cup_{m=1}^{M} \mathcal{S}_{m}=\Theta$, and

Preprint submitted to IEEE Transactions on Automatic Control. Received: January 8, 2011 11:25:21 PST 
$\operatorname{int} \mathcal{S}_{m} \neq \emptyset$, i.e., the triangles form a (nonoverlapping) partitioning of $\Theta$ and have nonempty interiors.

Step 2 Define

$$
\bar{A}_{\sigma, l}:=\tilde{A}_{\sigma, \tilde{h}_{l}, \tilde{\tau}_{l}}
$$

for all $\sigma \in\{1, \ldots, N\}$ and $\left(\tilde{h}_{l}, \tilde{\tau}_{l}\right) \in \mathcal{G}, l \in$ $\{1, \ldots, L\}$.

Step 3 To bound the approximation error, first construct the matrix $\bar{\Lambda}$, that, depending on the NCS model defined in Section II-A, is given by

$\bar{\Lambda}=\left\{\begin{aligned} \operatorname{diag}\left(A^{p}, A^{c}\right), & \text { if (12) is as in Section II-A1, } \\ \operatorname{diag}\left(A^{p}, 0\right), & \text { if (12) is as in Section II-A2, } \\ {\left[\begin{array}{cc}A^{p} & B^{p} C^{c} \\ 0 & A^{c}\end{array}\right], } & \text { if (12) is as in Section II-A3 }\end{aligned}\right.$

Decompose the matrix $\bar{\Lambda}$ into its real Jordan form [46], i.e., $\bar{\Lambda}:=T \Lambda T^{-1}$, where $T$ is an invertible matrix and

$$
\Lambda=\operatorname{diag}\left(\Lambda_{1}, \ldots, \Lambda_{K}\right)
$$

with $\Lambda_{i} \in \mathbb{R}^{n_{i} \times n_{i}}, i \in\{1, \ldots, K\}$, the $i$-th real Jordan block of $\bar{\Lambda}$.

Step 4 Compute for each real Jordan block $\Lambda_{i}, i \in$ $\{1, \ldots, K\}$, the worst-case approximation error of all triangles $\mathcal{S}_{m} \in \mathcal{H}, m \in\{1, \ldots, M\}$, i.e.,

$$
\begin{aligned}
\delta_{i}^{A} & =\max _{m \in\{1, \ldots, M\}} \max _{\sum_{j=1}^{3} \tilde{\alpha}^{j}=1, \tilde{\alpha}^{j} \geqslant 0} \tilde{\delta}_{i, m, \tilde{\alpha}}^{A}, \\
\delta_{i}^{E_{h}} & =\max _{m \in\{1, \ldots, M\}} \max _{\sum_{j=1}^{3} \tilde{\alpha}^{j}=1, \tilde{\alpha}^{j} \geqslant 0} \tilde{\delta}_{i, m, \tilde{\alpha}}^{E_{h}}, \\
\delta_{i}^{E_{h-\tau}} & =\max _{m \in\{1, \ldots, M\}} \max _{\sum_{j=1}^{3} \tilde{\alpha}^{j}=1, \tilde{\alpha}^{j} \geqslant 0} \tilde{\delta}_{i, m, \tilde{\alpha}}^{E_{h}-\tau},
\end{aligned}
$$

in which $\tilde{\alpha}=\left[\begin{array}{ccc}\tilde{\alpha}^{1} & \tilde{\alpha}^{2} & \tilde{\alpha}^{3}\end{array}\right]^{\top}$ and

$$
\begin{aligned}
& \tilde{\delta}_{i, m, \tilde{\alpha}}^{A}=\left\|e^{\Lambda_{i} \sum_{j=1}^{3} \tilde{\alpha}^{j} \tilde{h}_{l_{j}^{m}}^{m}}-\sum_{j=1}^{3} \tilde{\alpha}^{j} e^{\Lambda_{i} \tilde{h}_{l}^{m}}\right\|, \\
& \tilde{\delta}_{i, m, \tilde{\alpha}}^{E_{h}}=\left\|\sum_{j=1}^{3} \tilde{\alpha}^{j} \int_{\tilde{h}_{l_{j}^{m}}}^{\sum_{j=1}^{3} \tilde{\alpha}^{j} \tilde{h}_{l_{j}^{m}}} e^{\Lambda_{i} s} d s\right\|, \\
& \tilde{\delta}_{i, m, \tilde{\alpha}}^{E h-\tau}=\left\|\sum_{j=1}^{3} \tilde{\alpha}^{j} \int_{\tilde{h}_{l_{j}^{m}}-\tilde{\tau}_{l_{j}^{m}}}^{\sum_{j=1}^{3} \tilde{\alpha}^{j}\left(\tilde{h}_{l_{j}^{m}}-\tilde{\tau}_{l_{j}^{m}}\right)} e^{\Lambda_{i} s} d s\right\| .
\end{aligned}
$$

For a detailed explanation of the origin of the approximation error bounds, see the proof of Theorem III.2.

Step 5 Define

$$
\bar{C}_{\sigma}:=\left[\begin{array}{cc}
T^{-1} & 0 \\
T^{-1} B D C & T^{-1} B D \\
0 & -T^{-1} B \Gamma_{\sigma}
\end{array}\right]
$$

and

$$
\begin{array}{r}
\bar{B}:=\left[\begin{array}{ccc}
T & T & T \\
-C T & -C T & -C T
\end{array}\right] \cdot \operatorname{diag}\left(\delta_{1}^{A} I_{1}, \ldots, \delta_{K}^{A} I_{K},\right. \\
\\
\left.\quad \delta_{1}^{E_{h}} I_{1}, \ldots, \delta_{K}^{E_{h}} I_{K}, \delta_{1}^{E_{h-\tau}} I_{1}, \ldots, \delta_{K}^{E_{h-\tau}} I_{K}\right)
\end{array}
$$

with $I_{i}$ the identity matrix of size $n_{i}$, complying with the $i$-th real Jordan Block, and compute

$$
\varepsilon=\|\bar{B}\| \max _{\sigma \in\{1, \ldots, N\}}\left\{\left\|\bar{C}_{\sigma}\right\|\right\} .
$$

Step 6 In case that $\varepsilon>\varepsilon_{u}$, meaning that the user-specified tightness of the overapproximation in the sense of (30) is not achieved we add a pair $\left(\tilde{h}_{L+1}, \tilde{\tau}_{L+1}\right) \in \Theta$ to $\mathcal{G}$. In order to determine the specific pair to be added, compute the point $(h, \tau) \in \mathcal{S}_{m}$, where the maximum approximation error is achieved by solving

$$
\left(m^{\star}, \tilde{\alpha}^{\star}\right) \in \arg \max _{\substack{m \in\{1, \ldots, M\}, \sum_{j=1}^{3}=\tilde{\alpha}^{j}=1, \tilde{\alpha}^{j} \geqslant 0}} \tilde{\delta}_{i^{\star}, m, \tilde{\alpha}}^{j^{\star}}
$$

in which

$$
\left(i^{\star}, j^{\star}\right) \in \arg \max _{\substack{i \in\{1, \ldots, K\}, j \in\left\{A, E_{h}, E_{h}-\tau\right\}}}\left\{\delta_{i}^{j}\right\},
$$

and add this new pair $\left(\tilde{h}_{L+1}, \tilde{\tau}_{L+1}\right)=$ $\sum_{j=1}^{3} \tilde{\alpha}^{\star j}\left(\tilde{h}_{l_{m^{*}}^{j}}, \tilde{\tau}_{l_{m^{\star}}^{j}}\right)$ to the set $\mathcal{G}$, i.e., update $\mathcal{G}$ according to

$$
\mathcal{G}:=\mathcal{G} \cup\left\{\left(\tilde{h}_{L+1}, \tilde{\tau}_{L+1}\right)\right\},
$$

and redefine $L:=L+1$. Furthermore, subdivide the corresponding triangle $\mathcal{S}_{m^{\star}}$ into smaller triangles and replace $\mathcal{S}_{m^{\star}}$ by the smaller triangles in the set $\mathcal{H}$, i.e.,

$$
\begin{aligned}
\mathcal{H} & :=\left(\mathcal{H} \backslash \mathcal{S}_{m^{\star}}\right) \\
& \cup \operatorname{co}\left\{\left(\tilde{h}_{L+1}, \tilde{\tau}_{L+1}\right),\left(\tilde{h}_{l_{1}^{m}}, \tilde{\tau}_{l_{1}^{m}}\right),\left(\tilde{h}_{l_{2}^{m}}, \tilde{\tau}_{l_{2}^{m}}\right)\right\} \\
& \cup \operatorname{co}\left\{\left(\tilde{h}_{L+1}, \tilde{\tau}_{L+1}\right),\left(\tilde{h}_{l_{1}^{m}}, \tilde{\tau}_{l_{1}^{m}}\right),\left(\tilde{h}_{l_{3}^{m}}, \tilde{\tau}_{l_{3}^{m}}\right)\right\} \\
& \cup \operatorname{co}\left\{\left(\tilde{h}_{L+1}, \tilde{\tau}_{L+1}\right),\left(\tilde{h}_{l_{2}^{m}}, \tilde{\tau}_{l_{2}^{m}}\right),\left(\tilde{h}_{l_{3}^{m}}, \tilde{\tau}_{l_{3}^{m}}\right)\right\},
\end{aligned}
$$

redefine $^{1} M:=M+2$, and repeat the procedure from Step 2.

Step 7 In case $\varepsilon \leqslant \varepsilon_{u}$, the user-specified tightness of the overapproximation is achieved and the resulting additive uncertainty set $\boldsymbol{\Delta} \subseteq \mathbb{R}^{3\left(n_{p}+n_{c}\right) \times 3\left(n_{p}+n_{c}\right)}$ is given by

$$
\begin{gathered}
\boldsymbol{\Delta}=\left\{\operatorname{diag}\left(\Delta^{1}, \ldots, \Delta^{3 K}\right) \mid \Delta^{i+j L} \in \mathbb{R}^{n_{i} \times n_{i}},\right. \\
\left.\left\|\Delta^{i+j L}\right\| \leqslant 1, i \in\{1, \ldots, K\}, j \in\{0,1,2\}\right\} .
\end{gathered}
$$

Theorem III.2 Consider the NCS given by (12) where $\left(h_{k}, \tau_{k}\right) \in \Theta, k \in \mathbb{N}$, with $\Theta$ as in (5). If system (25) is obtained by following Procedure III.1 for some user-specified $\varepsilon_{u}>0$, then (27) holds and thus (25) is an overapproximation of (12). Furthermore, the overapproximation is $\varepsilon$-tight, in the sense that (30) holds, with $\varepsilon$ given by (39) and $\varepsilon \leqslant \varepsilon_{u}$.

Proof: The proof is given in the Appendix.

Remark III.3 In the special case that $\underline{h}=\bar{h}$ or that $\underline{\tau}=\bar{\tau}$, Procedure III.1 has to be modified slightly. This is because we proposed to form triangles $\mathcal{S}_{m}, m \in\{1, \ldots, M\}$ having the property that $\operatorname{int} \mathcal{S}_{m} \neq \emptyset$, which is not possible when $\underline{h}=\bar{h}$ or $\underline{\tau}=\bar{\tau}$. Instead, in this case, we partition $\Theta$

\footnotetext{
${ }^{1}$ In case one of the smaller triangles satisfies $\operatorname{int} \operatorname{co}\left\{\left(\tilde{h}_{L+1}, \tilde{\tau}_{L+1}\right),\left(\tilde{h}_{l_{i}^{m}}, \tilde{\tau}_{l_{i}^{m}}\right),\left(\tilde{h}_{l_{j}^{m}}, \tilde{\tau}_{l_{j}^{m}}\right)\right\}=\emptyset$ for some $i, j \in\{1,2,3\}$, meaning that $\left(\tilde{h}_{L+1}, \tilde{\tau}_{L+1}\right)$ lies on one of the edges of $\mathcal{S}_{m^{\star}}$, then this triangle is not added to the set $\mathcal{H}$, and the number of triangles in the partitioning increases according $M:=M+1$.
} 
into $M$ line-segments $\mathcal{S}_{1}, \ldots, \mathcal{S}_{M}$, such that, for each $\mathcal{S}_{m}$, $m \in\{1, \ldots, M\}$, it holds that

$$
\mathcal{S}_{m}=\operatorname{co}\left\{\left(\tilde{h}_{l_{1}^{m}}, \tilde{\tau}_{l_{1}^{m}}\right),\left(\tilde{h}_{l_{2}^{m}}, \tilde{\tau}_{l_{2}^{m}}\right)\right\},
$$

where $\left(\tilde{h}_{l_{j}^{m}}, \tilde{\tau}_{l_{j}^{m}}\right), j \in\{1,2\}$, now denote the vertices of the line segment $\mathcal{S}_{m}$. All other properties of $\mathcal{S}_{m}, m \in\{1, \ldots, M\}$ still hold and the remainder of the procedure can be applied mutatis mutandis.

UGES of the NCS system given by (1), (2a) or (2b), (3), and (7), with protocols satisfying (18) or (21), with $\left(h_{k}, \tau_{k}\right) \in \Theta$, $k \in \mathbb{N}$, can now be guaranteed by proving UGES of (25), with switching sequences satisfying (18) or (21), $\alpha_{k} \in \mathcal{A}$, and $\Delta_{k} \in \boldsymbol{\Delta}, k \in \mathbb{N}$, using the result of Lemma II.4 and the fact that (25) is a (tight) overapproximation of (12).

\section{Stability of Switched Systems With PARAMETRIC UNCERTAINTY}

In the previous sections, we discussed the NCS model and introduced a way to overapproximate it by a switched polytopic system with norm-bounded uncertainty. Given this switched uncertain system, we can analyse whether a switching sequence, as induced by a protocol, renders the switched system UGES.

We will start with so-called quadratic protocols that include the well-known TOD protocol as a particular case. The analysis is based on extensions of the ideas in [47], in which only switched linear systems without any form of uncertainty are considered. Hence, generalisations are needed to include switched polytopic systems with norm-bounded uncertainties as in (25). After the stability analysis for the quadratic protocols and the TOD protocol as a special case, we will also show how we can analyse stability for periodic protocols, having the RR protocol as a special case.

For proving stability of system (25), with switching sequences satisfying (18) or (21), we will employ the so-called full block S-procedure [48], which is applied in the following lemma.

Lemma IV.1 Let $\bar{P}$ be given and consider the set $\Delta$ as in (44). Then, it holds that

$$
\left[\begin{array}{c}
I \\
\bar{A}+\bar{B} \Delta \bar{C}
\end{array}\right]^{\top} \bar{P}\left[\begin{array}{c}
I \\
\bar{A}+\bar{B} \Delta \bar{C}
\end{array}\right] \prec 0 .
$$

for all $\Delta \in \Delta$, if

$$
\left[\begin{array}{ll}
I & 0 \\
\bar{A} & \bar{B}
\end{array}\right]^{\top} \bar{P}\left[\begin{array}{cc}
I & 0 \\
\bar{A} & \bar{B}
\end{array}\right]+\left[\begin{array}{cc}
0 & I \\
\bar{C} & 0
\end{array}\right]^{\top}\left[\begin{array}{cc}
-R & 0 \\
0 & R
\end{array}\right]\left[\begin{array}{cc}
0 & I \\
\bar{C} & 0
\end{array}\right] \prec 0
$$

where

$$
\begin{array}{r}
R \in \mathcal{R}=\left\{\operatorname { d i a g } \left(r_{1} I_{1}, \ldots, r_{K} I_{K}, r_{K+1} I_{1}, \ldots,\right.\right. \\
\left.r_{2 K} I_{K}, r_{2 K+1} I_{1}, \ldots, r_{3 K} I_{K}\right) \\
\left.\in \mathbb{R}^{3\left(n_{p}+n_{c}\right) \times 3\left(n_{p}+n_{c}\right)} \mid r_{i}>0\right\},
\end{array}
$$

where $I_{i}$ is an identity matrix of size $n_{i}$.

Proof: It follows directly from the full-block S-procedure [48] and the block-diagonal structure of (44).

By choosing a suitable $\bar{P}$, (46) can lead to a sufficient condition for stability of (25).

\section{A. Quadratic Protocols}

In this section, we assume that the switching function is given by (18). To analyse the stability of (25) having this switching function, we introduce the non-quadratic Lyapunov function

$$
V\left(\bar{x}_{k}\right)=\min _{i=1, \ldots, N} \bar{x}_{k}^{\top} P_{i} \bar{x}_{k}=\min _{\nu \in \mathcal{N}} \bar{x}_{k}^{\top} \sum_{i=1}^{N} \nu_{i} P_{i} \bar{x}_{k},
$$

where

$$
\mathcal{N}:=\left\{\nu \in \mathbb{R}^{N} \mid \sum_{i=1}^{N} \nu_{i}=1, \nu_{i} \geqslant 0, i \in\{1, \ldots, N\}\right\} .
$$

Furthermore, we introduce the class $\mathcal{M}$ of so-called Metzler matrices $\Pi=\left\{\pi_{j i}\right\}$ given by

$$
\begin{aligned}
& \mathcal{M}:= \\
& \left\{\Pi \in \mathbb{R}^{N \times N} \mid \sum_{j=1}^{N} \pi_{j i}=1, \pi_{j i} \geqslant 0, i, j \in\{1, \ldots, N\}\right\} .
\end{aligned}
$$

The main result of this section is presented in the following theorem.

Theorem IV.2 Assume that there exist a matrix $\Pi=\left\{\pi_{j i}\right\} \in$ $\mathcal{M}$, positive definite matrices $P_{i}$, and matrices $R_{i, l} \in \mathcal{R}, i \in$ $\{1, \ldots, N\}$ and $l \in\{1, \ldots, L\}$, satisfying

$$
\left[\begin{array}{cccc}
P_{i} & 0 & \bar{A}_{i, l}^{\top} \sum_{j=1}^{N} \pi_{j i} P_{j} & \bar{C}_{i}^{\top} R_{i, l} \\
\star & R_{i, l} & \bar{B}^{\top} \sum_{j=1}^{N} \pi_{j i} P_{j} & 0 \\
\star & \star & \sum_{j=1}^{N} \pi_{j i} P_{j} & 0 \\
\star & \star & \star & R_{i, l}
\end{array}\right] \succ 0,
$$

for all $i \in\{1, \ldots, N\}$ and $l \in\{1, \ldots, L\}$. Then, the switching law (18) renders the system (25) UGES. Consequently, the NCS given by (1), (2a) or (2b), (3), and (7) is also UGES if the switching law (18) is employed as the protocol.

Proof: The proof is given in the Appendix.

Remark IV.3 The results of Theorem IV.2 can be exploited in two ways: (i) For the design of a stabilising protocol. Then the conditions in (52) are not LMIs, but Bilinear Matrix Inequalities (BMIs) due to the presence of the product of $\pi_{j i}$ and $P_{j}$. Although literature on solving BMIs is available, see, e.g., [49]-[51], solving BMIs is considered to be of a high numerical complexity. (ii) Stability analysis for a given protocol. In the situation that the matrices $P_{i}, i \in\{1, \ldots, N\}$, are completely given for a particular quadratic protocol, the conditions (52) are LMIs in $\Pi \in \mathcal{M}$ and $R_{i, l} \in \mathcal{R}$, for all $i \in\{1, \ldots, N\}$ and $l \in\{1, \ldots, L\}$.

\section{B. The TOD Protocol}

In Section II-B, we showed that by suitable choice of $P_{i}, i \in$ $\{1, \ldots, N\}$, as in (19), the TOD protocol is a specific quadratic protocol. We can therefore use the result of Theorem IV.2 to determine the allowable range of transmission intervals and 
transmission delays of the NCS using the TOD protocol. This result is formalised in the following corollary, in which

$$
\begin{aligned}
& \overline{\mathcal{M}}:=\left\{\operatorname{diag}\left(0, \sum_{j=1}^{N} \pi_{j i} \Gamma_{j}\right)\right. \in \mathbb{R}^{n \times n} \mid \sum_{j=1}^{N} \pi_{j i}=1, \\
&\left.\pi_{j i} \geqslant 0, i, j \in\{1, \ldots, N\}\right\} .
\end{aligned}
$$

Corollary IV.4 Assume that there exist matrices $\bar{\Pi}_{i} \in \overline{\mathcal{M}}, i \in$ $\{1, \ldots, N\}$, a matrix $P$, matrices $R_{i, l} \in \mathcal{R}, i \in\{1, \ldots, N\}$ and $l \in\{1, \ldots, L\}$, satisfying

$$
\left[\begin{array}{cccc}
P-\operatorname{diag}\left(0, \Gamma_{i}\right) & 0 & \bar{A}_{i, l}^{\top}\left(P-\Pi_{i}\right) & \bar{C}_{i}^{\top} R_{i, l} \\
\star & R_{i, l} & \bar{B}^{\top}\left(P-\Pi_{i}\right) & 0 \\
\star & \star & P-\Pi_{i} & 0 \\
\star & \star & \star & R_{i, l}
\end{array}\right] \succ 0,
$$

for all $i \in\{1, \ldots, N\}$ and $l \in\{1, \ldots, L\}$, with $\Gamma_{i}$, as in (4). Then, the system (25) with (20) is UGES. Consequently, the NCS, given by (1), (2a) or (2b), (3), and (7), with the TOD protocol (20) is also UGES.

Proof: The proof follows directly from Theorem IV.2 and the fact that $P_{i}$ is structured as in (19). Therefore, it holds that $\sum_{j=1}^{N} \pi_{j i} P_{j}=P-\operatorname{diag}\left(0, \sum_{j=1}^{N} \pi_{j i} \Gamma_{j}\right)=: P-\Pi_{i}$.

\section{Periodic protocols and the RR Protocol}

We will now analyse another class of communication protocols, namely the periodic protocols, with the RR protocol as a special case. Hence, we need to analyse stability of the system (25) with a switching sequence induced by (21) or (22). This system is essentially a $\tilde{N}$-periodic uncertain system. For this system, we introduce positive definite matrices $P_{i}$, $i \in\{1, \ldots, N\}$, and a time-dependent periodic Lyapunov function given by

$$
V_{k}\left(\bar{x}_{k}\right)=\bar{x}_{k}^{\top} P_{k} \bar{x}_{k}, \quad \text { and } \quad V_{k+\tilde{N}}\left(\bar{x}_{k+\tilde{N}}\right)=V_{k}\left(\bar{x}_{k}\right) .
$$

We can now present the main result of this section.

Theorem IV.5 Assume that there exist positive definite matrices $P_{i}, i \in\{1, \ldots, \tilde{N}\}$, and matrices $R_{i, l} \in \mathcal{R}, i \in$ $\{1, \ldots, N\}$ and $l \in\{1, \ldots, L\}$, satisfying

$$
\left[\begin{array}{cccc}
P_{i} & 0 & \bar{A}_{\sigma_{i, l}}^{\top} P_{i+1} & \bar{C}_{\sigma_{i}}^{\top} R_{i, l} \\
\star & R_{i, l} & \bar{B}^{\top} P_{i+1} & 0 \\
\star & \star & P_{i+1} & 0 \\
\star & \star & \star & R_{i, l}
\end{array}\right] \succ 0
$$

where $P_{\tilde{N}+1}:=P_{1}$, for all $i \in\{1, \ldots, \tilde{N}\}$ and $l \in$ $\{1, \ldots, L\}$. Then, the system (25) with (22) is UGES and consequently, the NCS as given by (1), (2a) or (2b), (3), and (7) with a periodic protocol (21) is UGES.

Proof: The proof follows the same lines of reasoning as the proof of Theorem IV.2.

\section{NONCONSERVATIVENESS OF THE OVERAPPROXIMATION}

Given the results of the previous sections, it is now natural to ask if and how conservative the presented methodology is. The answer is given by the following result, showing that if the original system (12) (without any overapproximation), with protocol (18) or (21), is UGES in the sense that a Lyapunov function of a particular type exists, given by (49) or (55), respectively, the presented procedure based on the overapproximation will guarantee stability and will find a respective Lyapunov function, given that the overapproximation of (12) is sufficiently tight, i.e., (30) holds for a sufficiently small $\varepsilon>0$. Therefore, making a convex overapproximation, according to Procedure III.1, introduces no conservatism in the stability analysis as presented in the previous section.

In the following theorem, we will show the result for the NCS model (12) with protocol (18). A similar result holds for the NCS model (12) with protocol (21).

Theorem V.1 Suppose system (12), with protocol (18), has a Lyapunov function of the form (49), i.e., there exist a matrix $\Pi=\left\{\pi_{j i}\right\} \in \mathcal{M}$ and positive definite matrices $P_{i}$, $i \in\{1, \ldots, N\}$, such that

$$
\tilde{A}_{i, h, \tau}^{\top} \sum_{j=1}^{N} \pi_{j i} P_{j} \tilde{A}_{i, h, \tau}-P_{i} \preceq-\gamma I,
$$

for all $i \in\{1, \ldots, N\}$ and $(h, \tau) \in \Theta$, and some $\gamma>0$. Then, there exists an $\varepsilon_{0}$, such that for any $\varepsilon$-tight overapproximation satisfying (30), with $0<\varepsilon \leqslant \varepsilon_{0}$, the conditions of Theorem IV.2 hold.

Proof: The proof is given in the Appendix.

This result states that the convex overapproximation does not introduce conservatism when analysing UGES using mode-dependent quadratic Lyapunov functions.

\section{Illustrative EXAMPLE}

In this section, we illustrate the presented theory using a well-known benchmark example in the NCS literature, see, e.g., [25], [37]-[39], [41], consisting of a model of a batch reactor. The linearised batch reactor is given by (1), with

$$
\left[\begin{array}{l|l}
A^{p} & B^{p} \\
\hline C^{p} &
\end{array}\right]=\left[\begin{array}{rrrr|rr}
1.380 & -0.208 & 6.715 & -5.676 & 0 & 0 \\
-0.581 & -4.290 & 0 & 0.675 & 5.679 & 0 \\
1.067 & 4.273 & -6.654 & 5.893 & 1.136 & -3.146 \\
0.048 & 4.273 & 1.343 & -2.104 & 1.136 & 0 \\
\hline 1 & 0 & 1 & -1 & & \\
0 & 1 & 0 & 0 & &
\end{array}\right] .
$$

The continuous-time controller considered in [25], [37]-[39], [41] is given by (2a), with

$$
\left[\begin{array}{c|c}
A^{c} & B^{c} \\
\hline C^{c} & D^{c}
\end{array}\right]=\left[\begin{array}{cc|cc}
0 & 0 & 0 & 1 \\
0 & 0 & 1 & 0 \\
\hline-2 & 0 & 0 & -2 \\
0 & 8 & 5 & 0
\end{array}\right] .
$$

First, we will analyse the continuous-time NCS as also used in [37]-[39], [41]. As done in these references, we consider the TOD and RR protocol and assume that the controller is directly connected to the actuator, i.e., only the two outputs are transmitted via the network. Since communication delays 
are only considered in [41], and gives in absence of delays (i.e., $\underline{\tau}=\bar{\tau}=0$ ) the same results as in [37], we compare our results with [41]. This will show that our results provide significantly less conservative bounds on the uncertain transmission intervals and transmission delays than earlier results in the literature. Secondly, we illustrate that our framework can equally well deal with discrete-time controllers, a larger number of nodes than used in previous examples in the literature, and a nonzero lower bound on the transmission interval.

\section{A. Continuous-Time Controller}

In order to assess the bounds on the allowable transmission intervals and delays, we first define our NCS model as in Section II-A3. This model appropriately describes the situation as discussed in this example, where only the plant outputs $y$ are transmitted over the network and $u$ are sent continuously via a nonnetworked connection. Then, we derive the uncertain polytopic system (25) that overapproximates the NCS model (12), using Procedure III.1. As in [41], we try to obtain combinations of $\bar{h}$ and $\bar{\tau}$ for which the NCS is stable, and we assume that $\underline{\tau}=0$, and we take $\underline{h}=10^{-3}$. We cannot make $\underline{h}$ too small, because, by doing so, $\tilde{A}_{\sigma_{k}, h_{k}, \tau_{k}}$, as in (12), approaches the identity matrix and LMI solvers run into numerical problems, as the system becomes close to unstable. Note that [37]-[39], [41] also use nonzero lower bound on the transmission intervals to prevent Zeno behaviour, although, this lower bound can be taken arbitrarily small. Using Procedure III.1, we obtain a convex overapproximation, in which we choose $\varepsilon_{u}=3$ as decreasing $\varepsilon_{u}$ does not change the results in this example. Using the obtained overapproximation, we can check for which combinations of $\bar{h}$ and $\bar{\tau}$, the LMIs in Corollary IV.4 and Theorem IV.5 are feasible. This results for each $\bar{\tau}$ in the maximum achievable $\bar{h}$ (or vice versa) for which the LMIs in Corollary IV.4 and Theorem IV.5 are satisfied. This results in tradeoff curves, as shown in Fig. 3. These tradeoff curves can be used to impose or select a suitable network with a certain communication delay and a certain allowable transmission interval.

Moreover, in Fig 3, also the tradeoff curves as obtained in [41] are given. We conclude that our proposed methodology is less conservative than the one in [41]. More interestingly, in case there is no delay, i.e., $\underline{\tau}=\bar{\tau}=0$, the maximum allowable transmission interval $\bar{h}$ obtained in [37], which provide the least conservative results known in literature so far, was $\bar{h}=0.0108$, while we obtain $\bar{h}=0.0665$. In [39], $\bar{h}$ was estimated (using simulations) to be approximately 0.08 for the TOD protocol. Furthermore, for the RR protocol, [37] provides the bound $\bar{h}=0.009$ in the delay-free case, while we obtain $\bar{h}=0.0645$. Also in [39], for a constant transmission interval, i.e. $\underline{h}=\bar{h}$, the bound 0.0657 was obtained for the RR protocol. The case where the transmission interval is constant, provides an upper bound on the true maximum allowable transmission interval (MATI). We can therefore conclude that for this example, our methodology reduces conservatism significantly in comparison to existing methodologies and even approximates known estimates of the true MATI closely.

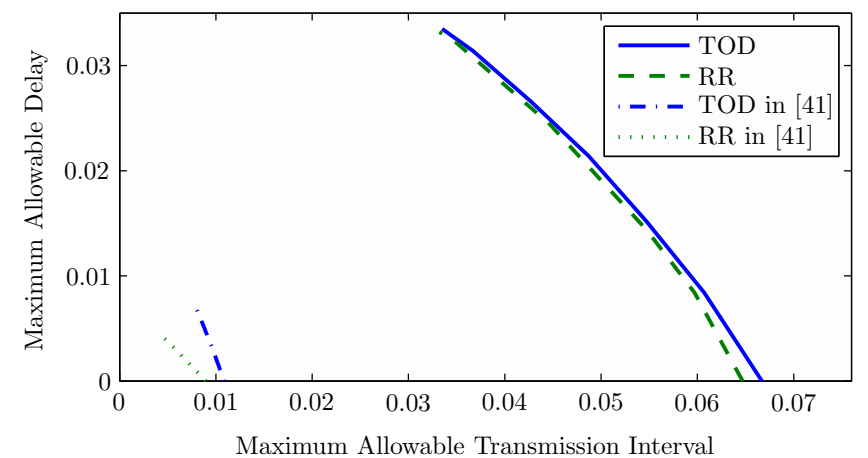

Fig. 3: Tradeoff curves between allowable transmission intervals and transmission delays for two different protocols.

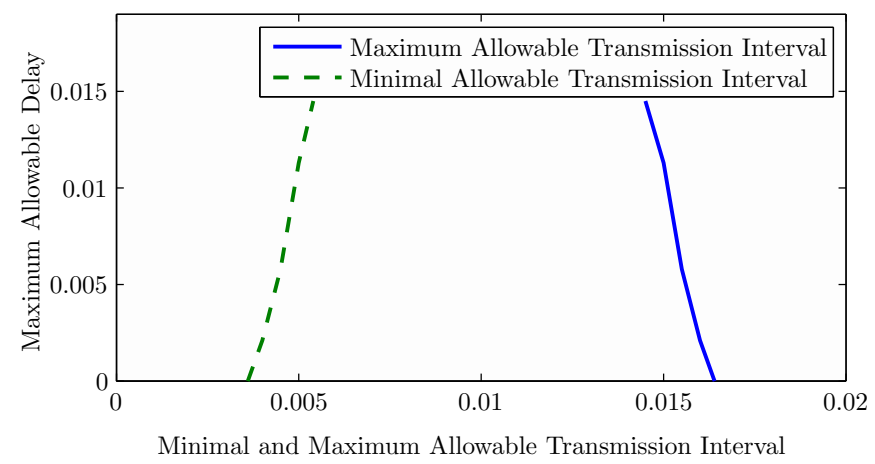

Fig. 4: Tradeoff curves between allowable transmission intervals and transmission delays.

\section{B. Discrete-Time Controller}

Next, we compute $\underline{h}, \bar{h}, \underline{\tau}$, and $\bar{\tau}$ for the NCS with a discrete-time controller as in (2b). Contrary to the example presented above, and all examples considered in [37]-[39], [41], we now designate a node to each single sensor and actuator, resulting in an NCS with four nodes. By doing so, we try to point out that our methodology is also suitable to study more complex problems. In this example, the controller is given by an exact discretisation of the continuous-time controller (2a) with matrices (59) using a zero-order hold and assuming a nominal transmission interval $h_{\text {nom }}=0.01$ and a bounded variation $h_{\mathrm{var}}$ around this nominal transmission interval. We assume that $\underline{\tau}=0$ and $\underline{h} \neq 0$, i.e., transmissions can be infinitely fast, but do not occur infinitely often. In this example, we select $\underline{h}=h_{\mathrm{nom}}-h_{\mathrm{var}}$ and $\bar{h}=h_{\mathrm{nom}}+h_{\mathrm{var}}$, where $2 h_{\mathrm{var}}>0$ determines the range of allowable transmission intervals and we only consider the RR protocol.

After obtaining a convex overapproximation using Procedure III.1, in which we have taken $\varepsilon_{u}=0.02$, and assessing stability using the results of Theorem IV.5, we can now plot for each $\bar{\tau}$, the largest range, determined by $\underline{h}=h_{\text {nom }}-h_{\text {var }}$ and $\bar{h}=h_{\text {nom }}+h_{\text {var }}$, for which UGES is guaranteed. In this example, we take $h_{\text {nom }}=0.01$, which results in the tradeoff curve as shown in Fig. 4.

\section{CONCLUSIONS}

In this paper, we studied the stability of Networked Control Systems (NCSs) that are subject to communication constraints, 
time-varying transmission intervals and time-varying delays. We analysed the stability of the NCS when the communication sequence is determined by one of the protocols in the newly introduced classes of quadratic protocols or periodic protocols, having the well-known Try-Once-Discard (TOD) and the Round-Robin (RR) as special cases. This analysis was based on a discrete-time switched linear uncertain system to model the NCS. A new and efficient convex overapproximation was proposed that allows us to analyse stability using a finite number of linear matrix inequalities. We presented an automated procedure to obtain the overapproximation and we formally showed that the convex overapproximation can be made arbitrarily tight and does not introduce conservatism. On a benchmark example, we illustrated the advantages and the effectiveness of the developed theory. In particular, we showed that stability can be guaranteed for a much larger maximum allowable transmission interval and maximum allowable transmission delay, when compared to the existing results in the literature. In addition, our results can be applied for stability analysis of NCS with discrete-time controllers and nonzero lower bounds on the transmission intervals and delays, which could not be analysed before even though they are highly relevant for practical implementations of networked controllers.

Future work focusses on studying the case where delays are not restricted to be smaller than the transmission interval, on the inclusion of quantisation effects of the sensor and actuator signals on the closed-loop stability and performance, and on co-design methods of the controller and the protocol.

\section{APPENDIX \\ PROOFS OF THEOREMS AND LEMMAS}

Proof of Lemma II.4: In Theorem 4 of [52], it was shown that a sampled-data system is UGES if and only if its corresponding discretised model is UGES and the intersample behaviour is so-called linearly uniformly globally bounded over $T$ (LUGBT), where $T$ is the sampling interval. This means, roughly speaking, that the intersample behaviour can be bounded by a linear function of the state of the system at the transmission instants. Since the discrete-time system is UGES by assumption, it only remains to show LUGBT. To do so, let us introduce an additional variable $\tilde{t}:=t-t_{k}$, for all $t \in\left(t_{k}, t_{k+1}\right]$. Solving the differential equation (1) on the interval $\tilde{t} \in\left(0, \tau_{k}\right]$ yields

$$
x^{p}\left(t_{k}+\tilde{t}\right)=e^{A^{p} \tilde{t}} x^{p}\left(t_{k}\right)+\int_{0}^{\tilde{t}} e^{A^{p}(\tilde{t}-s)} d s B^{p} \hat{u}\left(t_{k}\right)
$$

and on the interval $\tilde{t} \in\left(\tau_{k}, h_{k}\right]$

$$
\begin{aligned}
x^{p}\left(t_{k}+\tilde{t}\right)=e^{A^{p} \tilde{t}} x^{p}\left(t_{k}\right)+\int_{0}^{\tau_{k}} e^{A^{p}(\tilde{t}-s)} d s B^{p} \hat{u}\left(t_{k}\right) \\
+\int_{\tau_{k}}^{\tilde{t}} e^{A^{p}(\tilde{t}-s)} d s B^{p} \lim _{t \downarrow r_{k}} \hat{u}(t) .
\end{aligned}
$$

Or equivalently, when expressed in states at the sample instants, for $\tilde{t} \in\left(0, \tau_{k}\right]$,

$$
\begin{array}{rl}
x^{p}\left(t_{k}+\tilde{t}\right)=e^{A^{p} \tilde{t}} x_{k}^{p}+\int_{0}^{\tilde{t}} e^{A^{p} s} & d s B^{p}\left(D^{c} C^{p} x_{k}^{p}\right. \\
& \left.+C^{c} x_{k}^{c}+D^{c} e_{k}^{y}+e_{k}^{u}\right)
\end{array}
$$

and for $\tilde{t} \in\left(\tau_{k}, h_{k}\right]$,

$$
\begin{aligned}
x^{p}\left(t_{k}+\tilde{t}\right)= & e^{A^{p} \tilde{t}} x_{k}^{p}+\int_{0}^{\tilde{t}} e^{A^{p} s} d s B^{p}\left(D^{c} C^{p} x_{k}^{p}+C^{c} x_{k}^{c}\right. \\
& \left.+e_{k}^{u}+D^{c} e_{k}^{y}\right)-\int_{0}^{\tilde{t}-\tau_{k}} e^{A^{p} s} d s B^{p} \Gamma_{\sigma_{k}}^{u} e_{k}^{u} .
\end{aligned}
$$

Using (62) and (63), we can bound the intersample behaviour on the interval $\tilde{t} \in\left(0, h_{k}\right]$ by

$$
\begin{array}{r}
\left\|x^{p}\left(t_{k}+\tilde{t}\right)\right\| \leqslant\left\|e^{A^{p} \tilde{t}}\right\|\left\|x_{k}^{p}\right\|+\left\|\int_{0}^{\tilde{t}} e^{A^{p} s} d s B^{p}\right\|\left(\left\|C^{c}\right\|\left\|x_{k}^{c}\right\|\right. \\
\left.+\left\|D^{c} C^{p}\right\|\left\|x_{k}^{p}\right\|+\left\|D^{c}\right\|\left\|e_{k}^{y}\right\|+\left\|e_{k}^{u}\right\|\right) \\
+\left\|\int_{0}^{\tilde{t}-\tau_{k}} e^{A^{p} s} d s B^{p} \Gamma_{\sigma_{k}}^{u}\right\|\left\|e_{k}^{u}\right\|,
\end{array}
$$

Similar inequalities can be derived that bound the intersample behaviour for the state evolution $x^{c}(t)$ of (2a) and for the network-induced error given by (7). Therefore, by using the bounds on $h_{k}$ and $\tau_{k}$, the continuous-time NCS (1), (2a) or (2b), (3), and (7) is LUGBT. Consequently, Theorem 4 of [52] implies that the continuous-time NCS is UGES.

Proof of Theorem III.2: The proof is based on showing that Procedure III.1 yields that system (25) is an overapproximation of (12) in the sense that (27) holds, and that this overapproximation is tight in the sense that (30) holds for a $\varepsilon>0$, satisfying $\varepsilon \leqslant \varepsilon_{u}$ for some $\varepsilon_{u}$.

In order for (27) to hold, considering a fixed $\sigma \in$ $\{1, \ldots, N\}$, we should have that for all $(h, \tau) \in \Theta$, there exist an $\alpha \in \mathcal{A}$ and a $\Delta \in \boldsymbol{\Delta}$, such that (29) holds, i.e.,

$$
\tilde{A}_{\sigma, h, \tau}=\sum_{l=1}^{L} \alpha^{l} \bar{A}_{\sigma, l}+\bar{B} \Delta \bar{C}_{\sigma} .
$$

Therefore, given $L$ distinct pairs $\left(\tilde{h}_{l}, \tilde{\tau}_{l}\right) \in \Theta, l \in\{1, \ldots, L\}$, and $\bar{A}_{\sigma, l}$ as in (32), we can write the approximation error between $\tilde{A}_{\sigma, h, \tau}$ of (12) and $\sum_{l=1}^{L} \alpha^{l} \bar{A}_{\sigma, l}$ of (28) as

$$
\begin{aligned}
& \tilde{A}_{\sigma, h, \tau}-\sum_{l=1}^{L} \alpha^{l} \bar{A}_{\sigma, l}= \\
& \underbrace{\left[\begin{array}{ll}
T^{\top} & -(C T)^{\top} \\
T^{\top} & -(C T)^{\top} \\
T^{\top} & -(C T)^{\top}
\end{array}\right]^{\top}}_{=: \tilde{B}} \tilde{\Delta}_{\alpha, h, \tau} \underbrace{\left[\begin{array}{cc}
T^{-1} & 0 \\
T^{-1} B D C & T^{-1} B D \\
0 & -T^{-1} B \Gamma_{\sigma}
\end{array}\right]}_{=: \bar{C}_{\sigma}},
\end{aligned}
$$

where

$$
\begin{gathered}
\tilde{\Delta}_{\alpha, h, \tau}=\operatorname{diag}\left(T^{-1}\left(A_{h}-\sum_{l=1}^{L} \alpha^{l} A_{\tilde{h}_{l}}\right) T\right. \\
T^{-1}\left(E_{h}-\sum_{l=1}^{L} \alpha^{l} E_{\tilde{h}_{l}}\right) T \\
\left.T^{-1}\left(E_{h-\tau}-\sum_{l=1}^{L} \alpha^{l} E_{\tilde{h}_{l}-\tilde{\tau}_{l}}\right) T\right),
\end{gathered}
$$

in which $A_{h}, E_{h}, E_{h-\tau}$ are defined as in Section II-A1, II-A2 or II-A3. Using the real Jordan form of (33), we can observe 
that

$$
\begin{gathered}
T^{-1}\left(A_{h}-\sum_{l=1}^{L} \alpha^{l} A_{\tilde{h}_{l}}\right) T=e^{\Lambda h}-\sum_{l=1}^{L} \alpha^{l} e^{\Lambda \tilde{h}_{l}} \\
T^{-1}\left(E_{h}-\sum_{l=1}^{L} \alpha^{l} E_{\tilde{h}_{l}}\right) T=\sum_{l=1}^{L} \alpha^{l} \int_{\tilde{h}_{l}}^{h} e^{\Lambda s} d s \\
T^{-1}\left(E_{h-\tau}-\sum_{l=1}^{L} \alpha^{l} E_{\tilde{h}_{l}-\tilde{\tau}_{l}}\right) T=\sum_{l=1}^{L} \alpha^{l} \int_{\tilde{h}_{l}-\tilde{\tau}_{l}}^{h-\tau} e^{\Lambda s} d s
\end{gathered}
$$

hold for all $\alpha \in \mathcal{A}$ if $A_{h}, E_{h}$, and $E_{h-\tau}$ are defined as in Section II-A1 and II-A3. Since $E_{h}$ and $E_{h-\tau}$, when defined as in Section II-A2, contain identity matrices in the lowerright part, the left-hand side of (68b) and (68c) contain zero blocks. Therefore, in case of $E_{h}$ and $E_{h-\tau}$ being defined as in Section II-A2, equality in (68b) and (68c) do not automatically hold and we have to impose additional requirements on $\alpha \in$ $\mathcal{A}$ to ensure that the appropriate Jordan blocks of the righthand side of (68b) and (68c) also equal zero. These additional requirements are that

$$
\sum_{l=1}^{L} \alpha^{l} \tilde{h}_{l}=h, \quad \text { and } \quad \sum_{l=1}^{L} \alpha^{l}\left(\tilde{h}_{l}-\tilde{\tau}_{l}\right)=h-\tau,
$$

since substituting (69) into (68) indeed results in zero-blocks at the appropriate places in the left-hand side of (68). Now, combining (67) and (68) yields

$$
\tilde{\Delta}_{\alpha, h, \tau}=\sum_{l=1}^{L} \alpha^{l} \operatorname{diag}\left(e^{\Lambda h}-e^{\Lambda \tilde{h}_{l}}, \int_{\tilde{h}_{l}}^{h} e^{\Lambda s} d s, \int_{\tilde{h}_{l}-\tilde{\tau}_{l}}^{h-\tau} e^{\Lambda s} d s\right),
$$

provided that (69) holds.

As an intermediate step in the proof, we aim at finding a set $\tilde{\boldsymbol{\Delta}}$ of matrices, such that for all $(h, \tau) \in \Theta$ there is an $\alpha \in \mathcal{A}$ such that $\tilde{\Delta}_{\alpha, h, \tau} \in \tilde{\Delta}$. Since $\Theta=\cup_{m=1}^{M} \mathcal{S}_{m}$, we wil perform the construction of $\tilde{\boldsymbol{\Delta}}$ per triangle $\mathcal{S}_{m}, m \in\{1 \ldots, M\}$, and combine them later. Hence, for each $m \in\{1, \ldots, M\}$, we now aim at constructing $\tilde{\boldsymbol{\Delta}}^{m}$ such that for all $(h, \tau) \in \mathcal{S}_{m}$, $m \in\{1, \ldots, M\}$, there is an $\alpha \in \mathcal{A}$ such that $\tilde{\Delta}_{\alpha, h, \tau} \in \tilde{\boldsymbol{\Delta}}^{m}$. In particular, for $(h, \tau) \in \mathcal{S}_{m}, m \in\{1, \ldots, M\}$, with $\mathcal{S}_{m}$ as in (31), take $\bar{\alpha}_{j}^{l_{j}^{m}}=\tilde{\alpha}^{j}, j=\{1,2,3\}$, and $\bar{\alpha}^{i}=0, i \notin$ $\left\{l_{1}^{m}, l_{2}^{m}, l_{3}^{m}\right\}$, where $\sum_{j=1}^{3} \tilde{\alpha}^{j}\left(\tilde{h}_{j}^{m}, \tilde{\tau}_{j}^{m}\right)=(h, \tau), \sum_{j=1}^{3} \tilde{\alpha}^{j}=$ 1 , and $\tilde{\alpha}^{j} \geqslant 0, j \in\{1,2,3\}$. Let us now bound the norm of (70) for triangle $\mathcal{S}_{m}, m \in\{1, \ldots, M\}$, and per Jordan block $\Lambda_{i}, i \in\{1, \ldots, K\}$ using this particular choice $\bar{\alpha}$ for $\alpha$. Hence, for all $(h, \tau) \in \mathcal{S}_{m}, \tilde{\Delta}_{\bar{\alpha}, h, \tau} \in \tilde{\boldsymbol{\Delta}}^{m}$ with

$$
\begin{aligned}
& \tilde{\Delta}^{m}:= \\
& \left\{\operatorname{diag}\left(\tilde{\Delta}_{1}^{A}, \ldots, \tilde{\Delta}_{K}^{A}, \tilde{\Delta}_{1}^{E_{h}}, \ldots, \tilde{\Delta}_{K}^{E_{h}}, \tilde{\Delta}_{1}^{E_{h-\tau}}, \ldots, \tilde{\Delta}_{K}^{E_{h-\tau}}\right) \mid\right. \\
& \quad\left\|\tilde{\Delta}_{i}^{A}\right\| \leqslant \max _{\substack{\sum_{j=1}^{3} \tilde{\alpha}^{j}=1, \tilde{\alpha}^{j} \geqslant 0}} \tilde{\delta}_{i, m, \tilde{\alpha}}^{A},\left\|\tilde{\Delta}_{i}^{E_{h}}\right\| \leqslant \max _{\substack{\sum_{j=1}^{3} \tilde{\alpha}^{j}=1, \tilde{\alpha}^{j} \geqslant 0}} \tilde{\delta}_{i, m, \tilde{\alpha}}^{E_{h}}, \\
& \left.\left\|\tilde{\Delta}_{i}^{E_{h-\tau}}\right\| \leqslant \max _{\substack{\sum_{j=1}^{3} \tilde{\alpha}^{j}=1, \tilde{\alpha}^{j} \geqslant 0}} \tilde{\delta}_{i, m, \tilde{\alpha}}^{E_{h-\tau}}, i \in\{1, \ldots, K\}\right\}, \quad(71)
\end{aligned}
$$

for $m \in\{1, \ldots, M\}$ in which $\tilde{\delta}_{i, m, \tilde{\alpha}}^{A}, \tilde{\delta}_{i, m, \tilde{\alpha}}^{E_{h}}$, and $\tilde{\delta}_{i, m, \tilde{\alpha}}^{E_{h-\tau}}$ are given by (36). This upper bound on the approximation errors allows us to write

$$
\begin{aligned}
& \left\{\tilde{A}_{\sigma, h, \tau} \mid(h, \tau) \in \mathcal{S}_{m}\right\} \subseteq \\
& \left\{\sum_{j=1}^{3} \tilde{\alpha}^{j} \bar{A}_{\sigma, l_{j}^{m}}+\tilde{B} \tilde{\Delta}^{m} \bar{C}_{\sigma} \mid \sum_{j=1}^{3} \tilde{\alpha}^{j}=1, \tilde{\alpha}^{j} \geqslant 0,\right. \\
& \left.j \in\{1,2,3\}, \tilde{\Delta}^{m} \in \tilde{\Delta}^{m}\right\} .
\end{aligned}
$$

To obtain $\boldsymbol{\Delta}$ independent of $m$, as in (44), let us now introduce the scaling matrix

$$
\begin{aligned}
U:=\operatorname{diag}\left(\delta_{1}^{A} I_{1}, \ldots, \delta_{K}^{A} I_{K}, \delta_{1}^{E_{h}} I_{1}, \ldots, \delta_{K}^{E_{h}} I_{K},\right. \\
\\
\left.\delta_{1}^{E_{h-\tau}} I_{1}, \ldots, \delta_{K}^{E_{h-\tau}} I_{K}\right)
\end{aligned}
$$

in which $I_{i}$ is an identity matrix of size $n_{i}$, complying with the size of the $\tilde{\Delta}_{i}$ and observe that $\tilde{\boldsymbol{\Delta}}^{m} \subseteq U \boldsymbol{\Delta}$, with $\boldsymbol{\Delta}$ as in (44). Now due to (35) and (38), $\bar{B}=\tilde{B} \cdot U$, and this allows us to rewrite (72) as

$$
\begin{aligned}
& \left\{\tilde{A}_{\sigma, h, \tau} \mid(h, \tau) \in \mathcal{S}_{m}\right\} \\
& \subseteq\left\{\sum_{j=1}^{3} \tilde{\alpha}^{j} \bar{A}_{\sigma, l_{j}^{m}}+\bar{B} \Delta \bar{C}_{\sigma} \mid \sum_{j=1}^{3} \tilde{\alpha}^{j}=1, \tilde{\alpha}^{j} \geqslant 0,\right. \\
& j \in\{1,2,3\}, \Delta \in \boldsymbol{\Delta}\} \\
& \subseteq\left\{\sum_{l=1}^{L} \alpha^{l} \bar{A}_{\sigma, l}+\bar{B} \Delta \bar{C}_{\sigma} \mid \alpha \in \mathcal{A}, \Delta \in \boldsymbol{\Delta}\right\},
\end{aligned}
$$

with $\mathcal{A}$ as in (26) and $\boldsymbol{\Delta}$ as in (44), which is . By taking the convex hull over all $m \in\{1, \ldots, M\}$ in the left-hand-side and observing that the right-hand-side is independent of $m$, we obtain (27).

To show that (30) holds for $\varepsilon$ as in (39), we consider a fixed $\sigma \in\{1, \ldots, N\}$ and show that for all $\alpha \in \mathcal{A}$ and $\Delta \in \Delta$, there exist a pair $(h, \tau) \in \Theta$ and a $\bar{\Delta}$, satisfying $\|\bar{\Delta}\| \leqslant \varepsilon$, such that

$$
\begin{aligned}
& \sum_{l=1}^{L} \alpha^{l} \bar{A}_{\sigma, l}+\bar{B} \Delta \bar{C}_{\sigma} \\
& \quad \in \operatorname{co}\left\{\tilde{A}_{\sigma, h, \tau} \mid(h, \tau) \in \Theta\right\}+\{\bar{\Delta} \mid\|\bar{\Delta}\| \leqslant \varepsilon\} .
\end{aligned}
$$

Since by definition it holds that $\sum_{l=1}^{L} \alpha^{l} \bar{A}_{\sigma, l}=$ $\sum_{l=1}^{L} \alpha^{l} \bar{A}_{\sigma, \tilde{h}_{l}, \tilde{\tau}_{l}} \in \operatorname{co}\left\{\bar{A}_{\sigma, h, \tau} \mid(h, \tau) \in \Theta\right\}$, this inclusion is satisfied if

$$
\left\|\bar{B} \Delta \bar{C}_{\sigma}\right\| \leqslant \varepsilon,
$$

which holds for $\varepsilon \leqslant \varepsilon_{u}$ as in (39), due to the fact that Procedure III. 1 terminates not until $\varepsilon \leqslant \varepsilon_{u}$.

Proof of Theorem IV.2: The proof is based on showing that $V$ as in (49) is a Lyapunov function for the switched uncertain system (25) with switching law (18). Note that $V\left(\bar{x}_{k}\right)=\bar{x}_{k}^{\top} P_{i} \bar{x}_{k}$, with $i=\sigma_{k}$, due to (18). Now, we obtain 
using (49) and (25) that

$$
\begin{aligned}
& V\left(\bar{x}_{k+1}\right) \\
& =\min _{\nu \in \mathcal{N}} \bar{x}_{k+1}^{\top} \sum_{j=1}^{N} \nu_{j} P_{j} \bar{x}_{k+1} \\
& =\min _{\nu \in \mathcal{N}} \bar{x}_{k}^{\top}\left(\sum_{l_{1}=1}^{L} \alpha_{k}^{l_{1}} \bar{A}_{\sigma_{k}, l_{1}}+\bar{B} \Delta_{k} \bar{C}_{\sigma_{k}}\right)^{\top} \\
& \quad \sum_{j=1}^{N} \nu_{j} P_{j}\left(\sum_{l_{2}=1}^{L} \alpha_{k}^{l_{2}} \bar{A}_{\sigma_{k}, l_{2}}+\bar{B} \Delta_{k} \bar{C}_{\sigma_{k}}\right) \bar{x}_{k} \\
& \leqslant \bar{x}_{k}^{\top}\left(\sum_{l_{1}=1}^{L} \alpha_{k}^{l_{1}} \bar{A}_{i, l_{1}}+\bar{B} \Delta_{k} \bar{C}_{i}\right)^{\top} \\
& \quad \sum_{j=1}^{N} \pi_{j i} P_{j}\left(\sum_{l_{2}=1}^{L} \alpha_{k}^{l_{2}} \bar{A}_{i, l_{2}}+\bar{B} \Delta_{k} \bar{C}_{i}\right) \bar{x}_{k} .
\end{aligned}
$$

To obtain UGES, it is sufficient to require that the Lyapunov function is strictly decreasing in the sense that (due to (77))

$$
\begin{aligned}
\left(\sum_{l_{1}=1}^{L} \alpha_{k}^{l_{1}} \bar{A}_{i, l_{1}}+\bar{B} \Delta \bar{C}_{i}\right)^{\top} \sum_{j=1}^{N} \pi_{j i} P_{j} & \\
& \left(\sum_{l_{2}=1}^{L} \alpha_{k}^{l_{2}} \bar{A}_{i, l_{2}}+\bar{B} \Delta \bar{C}_{i}\right)-P_{i} \prec 0 .
\end{aligned}
$$

for all $\alpha \in \mathcal{A}, \Delta \in \overline{\boldsymbol{\Delta}}$, and $i \in\{1, \ldots, N\}$. Here, we used that $\boldsymbol{\Delta} \subseteq \overline{\boldsymbol{\Delta}}$, as stated in Lemma IV.1. By taking a Schur complement, realising that $\sum_{j=1}^{N} \pi_{j i} P_{j} \succ 0$, and using that $\alpha_{k} \in \mathcal{A}$, we obtain that (78) is equivalent to stating that

$$
\sum_{l=1}^{L} \alpha_{k}^{l} \underbrace{\left[\begin{array}{c}
P_{i} \\
\sum_{j=1}^{N} \pi_{j i} P_{j}\left(\bar{A}_{i, l}+\bar{B} \Delta \bar{C}_{i}\right)
\end{array} \begin{array}{c}
\left(\bar{A}_{i, l}+\bar{B} \Delta \bar{C}_{i}\right)^{\top} \sum_{j=1}^{N} \pi_{j i} P_{j} \\
\sum_{j=1}^{N} \pi_{j i} P_{j}
\end{array}\right]}_{G_{i, l}}
$$

is positive definite for all $\alpha \in \mathcal{A}, \Delta \in \overline{\boldsymbol{\Delta}}$, and $i \in\{1, \ldots, N\}$. A necessary and sufficient condition for positive definiteness of (79), for all $\alpha_{k} \in \mathcal{A}$, is that $G_{i, l} \succ 0$ for all $i \in\{1, \ldots, N\}$ and $l \in\{1, \ldots, L\}$. Using again a Schur complement, we can rewrite the condition $G_{i, l} \succ 0$ as follows:

$$
P_{i}-\left(\bar{A}_{i, l}+\bar{B} \Delta \bar{C}_{i}\right)^{\top} \sum_{j=1}^{N} \pi_{j i} P_{j}\left(\bar{A}_{i, l}+\bar{B} \Delta \bar{C}_{i}\right) \succ 0,
$$

or equivalently,

$$
\left[\begin{array}{c}
I \\
\bar{A}_{i, l}+\bar{B} \Delta \bar{C}_{i}
\end{array}\right]^{\top}\left[\begin{array}{cc}
-P_{i} & 0 \\
0 & \sum_{j=1}^{N} \pi_{j i} P_{j}
\end{array}\right]\left[\begin{array}{c}
I \\
\bar{A}_{i, l}+\bar{B} \Delta \bar{C}_{i}
\end{array}\right] \prec 0
$$

for all $\Delta \in \overline{\boldsymbol{\Delta}}, i \in\{1, \ldots, N\}$ and $l \in\{1, \ldots, L\}$. As (81) has the form of (46) of Lemma IV.1, it is therefore implied by (47). Applying a Schur complement yields (52) for all $i \in$ $\{1, \ldots, N\}$ and all $l \in\{1, \ldots, L\}$. Since (52) holds by the hypothesis of the theorem, we can conclude that $V$ is strictly decreasing in spite of the presence of the uncertainty. Standard Lyapunov-based stability arguments now prove that (25) with (18) is UGES. Using that (25) is an overapproximation of (12) as proven in Theorem III.2 and subsequently, using the result of Lemma II.4, it follows that the NCS system given by (1), (2a) or (2b), (3), and (7) is UGES.

Proof of Theorem V.1: Since (57) holds for all pairs $(h, \tau) \in \Theta$ and, therefore, for all $\left(\tilde{h}_{l}, \tilde{\tau}_{l}\right) \in \Theta, l \in\{1, \ldots, L\}$, we have that

$$
\left[\begin{array}{cc}
\bar{A}_{i, l}^{\top} \sum_{j=1}^{N} \pi_{j i} P_{j} \bar{A}_{i, l}-P_{i}+\gamma I & 0 \\
0 & 0
\end{array}\right] \preceq 0,
$$

for all $i \in\{1, \ldots, N\}$ and $l \in\{1, \ldots, L\}$. Note that (82) holds irrespective of the choice of $\left(\tilde{h}_{l}, \tilde{\tau}_{l}\right) \in \Theta, l \in\{1, \ldots, L\}$. Now suppose that we would establish that there exist matrices $R_{i, l} \in \mathcal{R}, i \in\{1, \ldots, N\}$ and $l \in\{1, \ldots, L\}$, such that

$$
\sum_{j=1}^{N} \pi_{j i}\left[\begin{array}{cc}
\bar{C}_{i}^{\top} R_{i, l} \bar{C}_{i}-\gamma I & \bar{A}_{i, l}^{\top} P_{j} \bar{B} \\
\bar{B}^{\top} P_{j} \bar{A}_{i, l} & \bar{B}^{\top} P_{j} \bar{B}-R_{i, l}
\end{array}\right] \prec 0,
$$

for all $i \in\{1, \ldots, N\}$ and $l \in\{1, \ldots, L\}$. Then, combining this expression with (82) yields, after taking a Schur complement, the conditions of Theorem IV.2. Hence, if the fact that (30) holds for a sufficiently small $\varepsilon$, implies that (83) holds for some $R_{i, l} \in \mathcal{R}$, we completed the proof.

Therefore, it remains to show that there exists an $\varepsilon_{0}$, such that for any $0<\varepsilon \leqslant \varepsilon_{0},(83)$ is satisfied for some $R_{i, l} \in \mathcal{R}$. Note that (83) holds if

$$
\bar{C}_{i}^{\top} R_{i, l} \bar{C}_{i}+\bar{A}_{i, l}^{\top} P_{j} \bar{B}\left(R_{i, l}-\bar{B}^{\top} P_{j} \bar{B}\right)^{-1} \bar{B}^{\top} P_{j} \bar{A}_{i, l} \prec \gamma I,
$$

and $\bar{B}^{\top} P_{j} \bar{B} \prec R_{i, l}$, for some $R_{i, l} \in \mathcal{R}$, and for all $i, j \in$ $\{1, \ldots, N\}$ and $l \in\{1, \ldots, L\}$. By choosing $R_{i, l}=r I$, for all $i \in\{1, \ldots, N\}$ and $l \in\{1, \ldots, L\}$ with $r>0$, we can observe that (84) is implied by

$$
\frac{\left\|P_{j} \bar{A}_{i, l}\right\|^{2}\|\bar{B}\|^{2}}{r-\lambda_{\min }\left(\bar{B}^{\top} P_{j} \bar{B}\right)}<\gamma-r\left\|\bar{C}_{i}\right\|^{2},
$$

where $\lambda_{\min }\left(\bar{B}^{\top} P_{j} \bar{B}\right)$ denotes the minimum eigenvalue of $\bar{B}^{\top} P_{j} \bar{B}$. Since it holds that $\lambda_{\min }\left(\bar{B}^{\top} P_{j} \bar{B}\right) \leqslant\|\bar{B}\|^{2}\left\|P_{j}\right\|$, (85) is implied by

$$
\left\|P_{j} \bar{A}_{i, l}\right\|^{2}\|\bar{B}\|^{2}<\left(\gamma-r\left\|\bar{C}_{i}\right\|^{2}\right)\left(r-\|\bar{B}\|^{2}\left\|P_{j}\right\|\right),
$$

Furthermore, $\bar{B}^{\top} P_{j} \bar{B} \prec R_{i, l}$ is implied by $\|\bar{B}\|^{2}\left\|P_{j}\right\|<r$, for some $r>0$ and all $i, j \in\{1, \ldots, N\}$ and $l \in\{1, \ldots, L\}$. Now choosing $r=\frac{\gamma}{2\left\|C_{i}\right\|^{2}}$, multiplying the left-hand and the righthand side of (86) by $\left\|\bar{C}_{i}\right\|^{2}$, and realising that $\|\bar{B}\|\left\|\bar{C}_{i}\right\| \leqslant \varepsilon$ yields that (86), and thereby (84), is satisfied if

$$
\varepsilon^{2}\left\|P_{j} \bar{A}_{i, l}\right\|^{2}<\frac{1}{4} \gamma^{2}-\frac{1}{2} \gamma \varepsilon^{2}\left\|P_{j}\right\|,
$$

and that $\bar{B}^{\top} P_{j} \bar{B} \prec R_{i, l}$ is satisfied if $\varepsilon^{2}\left\|P_{j}\right\|<\frac{1}{2} \gamma$, which can be satisfied by choosing $\varepsilon$ sufficiently small.

Therefore, if (49) is a Lyapunov function for system (12), with protocol (20), then there exists an $\varepsilon_{0}>0$, such that for any overapproximation satisfying (30) with $0<\varepsilon \leqslant \varepsilon_{0}$, the conditions of Theorem IV.2 hold, which completes the proof.

\section{REFERENCES}

[1] J. Hespanha, P. Naghshtabrizi, and Y. Xu, "A survey of recent results in networked control systems," Proc. IEEE, pp. 138-162, 2007.

[2] W. Zhang, M. Branicky, and S. Phillips, "Stability of networked control systems," IEEE Control Syst. Mag., vol. 21, pp. 84-99, 2001.

[3] Y. Tipsuwan and M.-Y. Chow, "Control methodologies in networked control systems," Control Eng. Pract., vol. 11, pp. 1099-1111, 2003.

[4] T. Yang, "Networked control system: a brief survey," IEE Proc. Control Theory \& Applications, vol. 153, pp. 403-412, 2006.

[5] R. Brockett and D. Liberzon, "Quantized feedback stabilization of linear systems," IEEE Trans. Autom. Control, vol. 45, pp. 1279-1289, 2000.

[6] D. Delchamps, "Stabilizing a linear system with quantized state feedback," IEEE Trans. Autom. Control, vol. 35, pp. 916-924, 1990.

[7] W. Heemels, H. Siahaan, A. Juloski, and S. Weiland, "Control of quantized linear systems: an $l_{1}$-optimal control approach," in Proc. American Control Conference, 2003, pp. 3502-3507. 
[8] D. Liberzon, "On stabilization of linear systems with limited information," IEEE Trans. Autom. Control, vol. 48, pp. 304-307, 2003.

[9] G. Nair and R. Evans, "Stabilizability of stochastic linear systems with finite feedback data rates," SIAM J. Control Optim., vol. 43, pp. 413436, 2004.

[10] X. Liu and A. Goldsmith, "Kalman filtering with partial observation losses," Proc. IEEE Conf. Decision \& Control, vol. 4, pp. 4180-4186, 2004.

[11] S. Smith and P. Seiler, "Estimation with lossy measurements: jump estimators for jump systems," IEEE Trans. Autom. Control, vol. 48, pp. 2163-2171, 2003

[12] B. Sinopoli, L. Schenato, M. Franceschetti, K. Poolla, M. Jordan, and S. Sastry, "Kalman filtering with intermittent observations," IEEE Trans. Autom. Control, vol. 49, pp. 1453-1464, 2004

[13] L. Montestruque and P. Antsaklis, "Stability of model-based networked control systems with time-varying transmission times," IEEE Trans. Autom. Control, vol. 49, pp. 1562-1572, 2004.

[14] Y. Suh, "Stability and stabilization of nonuniform sampling systems," Automatica, vol. 44, pp. 3222-3226, 2008

[15] H. Fujioka, "A discrete-time approach to stability analysis of systems with aperiodic sample-and-hold devices," IEEE Trans. Autom. Control, vol. 54, pp. 2440-2445, 2009.

[16] A. Balluchi, P. Murrieri, and A. L. Sangiovanni-Vincentelli, "Controller synthesis on non-uniform and uncertain discrete-time domains," in Proc. Conf. Hybrid Systems: Computation and Control, 2005, pp. 118-133.

[17] L. Hetel, J. Daafouz, and C. Iung, "Analysis and control of LTI and switched systems in digital loops via an event-based modeling," Int. J. Control, vol. 81, pp. 1125-1138, 2008.

[18] L. Dritsas and A. Tzes, "Robust stability analysis of networked systems with varying delays," Int. J. Control, vol. 82, p. 2347 2355, 2009.

[19] M. Cloosterman, N. van de Wouw, W. Heemels, and H. Nijmeijer, "Stability of networked control systems with uncertain time-varying delays," IEEE Trans. Autom. Control, vol. 54, pp. 1575 - 1580, 2009.

[20] R. Gielen, S. Olaru, M. Lazar, W. P. M. H. Heemels, N. van de Wouw, and S. Niculescu, "On polytopic inclusions as a modeling framework for systems with time-varying delays," Automatica, vol. 46, pp. 615-619, 2010.

[21] C.-Y. Kao and B. Lincoln, "Simple stability criteria for systems with time-varying delays," Automatica, vol. 40, pp. 1429-1434, 2004.

[22] P. Naghshtabrizi, J. Hespanha, and A. Teel, "Stability of delay impulsive systems with application to networked control systems," in Proc. American Control Conf., New York, USA, 2007, pp. 4899-4904.

[23] L. Zhang, Y. Shi, T. Chen, and B. Huang, "A new method for stabilization of networked control systems with random delays," IEEE Trans. Autom. Control, vol. 50, pp. 1177-1181, 2005.

[24] L. Hetel, J. Daafouz, and C. Iung, "Stabilization of arbitrary switched linear systems with unknown time-varying delays," IEEE Trans. Autom. Control, vol. 51, pp. 1668-1674, 2006.

[25] D. Dačić and D. Nešić, "Quadratic stabilization of linear networked control systems via simultaneous protocol and controller design," Automatica, vol. 43, pp. 1145-1155, 2007.

[26] R. Brockett, "Stabilization of motor networks," in Proc. IEEE Conf. Decision \& Control, vol. 2, 1995, pp. 1484-1488.

[27] D. Hristu and K. Morgansen, "Limited communication control," Syst. Control Lett., vol. 37, pp. 193-205, 1999.

[28] H. Rehbinder and M. Sanfridson, "Scheduling of a limited communication channel for optimal control," Automatica, vol. 40, pp. 491-500, 2004.

[29] D. Nešić and D. Liberzon, "A unified approach to controller design for systems with quantization and time scheduling," in Proc. IEEE Conf. Decision \& Control, 2007, pp. 3939-3944.

[30] M. Cloosterman, L. Hetel, N. Wouw, W. Heemels, J. Daafouz, and H. Nijmeijer, "Controller synthesis for networked control systems," Accepted for Automatica, 2010.

[31] D. Liberzon, "Quantization, time delays, and nonlinear stabilization," IEEE Trans. Autom. Control, vol. 51, pp. 1190-1195, 2006.

[32] M. Cloosterman, N. van de Wouw, W. Heemels, and H. Nijmeijer, "Stabilization of networked control systems with large delays and packet dropouts," in Proc. American Control Conf., 2008, pp. 4991-4996.

[33] M. Yu, L. Wang, T. Chu, and F. Hao, "An LMI approach to networked control systems with data packet dropout and transmission delays," Proc. IEEE Conf. Decision \& Control, vol. 4, pp. 3545-3550, 2004.

[34] P. Naghshtabrizi and J. Hespanha, "Stability of networked control systems with variable sampling and delay," in 44th Allerton Conf. Communications, Control \& Computing, 2006.
[35] N. van de Wouw, P. Naghshtabrizi, M. Cloosterman, and J. Hespanha, "Tracking control for sampled-data systems with uncertain sampling intervals and delays," Int. J. Robust \& Nonlinear Control, vol. 20, pp. $387-411,2010$.

[36] M. Donkers, L. Hetel, W. Heemels, N. van de Wouw, and M. Steinbuch, "Stability analysis of networked control systems using a switched linear systems approach," in Proc. Conf. Hybrid Systems: Computation and Control, 2009, pp. 150-164.

[37] D. Carnevale, A. Teel, and D. Nešić, "A Lyapunov proof of improved maximum allowable transfer interval for networked control systems," IEEE Trans. Autom. Control, vol. 52, pp. 892-897, 2007.

[38] D. Nešić and A. Teel, "Input-output stability properties of networked control systems," IEEE Trans. Autom. Control, vol. 49, pp. 1650-1667, 2004.

[39] G. Walsh, H. Ye, and L. Bushnell, "Stability analysis of networked control systems," IEEE Trans. Control Syst. Technology, vol. 10, pp. 438-446, 2002.

[40] G. Walsh, O. Belidman, and L. Bushnell, "Asymptotic behavior of nonlinear networked control systems," IEEE Trans. Autom. Control, vol. 46, pp. 1093-1097, 2001.

[41] W. Heemels, A. Teel, N. van de Wouw, and D. Nešić, "Networked control systems with communication constraints: Tradeoffs between transmission intervals, delays and performance," IEEE Trans. Autom. Control, 2010

[42] A. Chaillet and A. Bicchi, "Delay compensation in packet-switching networked controlled sytems," in Proc. IEEE Conf. Decision \& Control, 2008, pp. 3620-3625.

[43] M. Tabbara, D. Nešić, and A. Teel, "Stability of wireless and wireline networked control systems," IEEE Trans. Autom. Control, vol. 52, pp. 1615-1630, 2007.

[44] R. Goebel, R. Sanfelice, and A. Teel, "Hybrid dynamical systems," IEEE Control Syst. Mag., vol. 29, pp. 28-93, 2009.

[45] W. Heemels, N. Wouw, R. Gielen, M. Donkers, L. Hetel, S. Olaru, M. Lazar, J. Daafouz, and S. I. Niculescu, "Comparison of overapproximation methods for stability analysis of networked control systems," in Proc. Conf. Hybrid Systems: Computation and Control, 2010, pp. 181-190.

[46] R. Horn and C. R. Johnson, Matrix Analysis. Cambridge University Press, 1985.

[47] J. Geromel and P. Colaneri, "Stability and stabilization of discrete time switched systems," Int. J. Control, vol. 79, pp. 719-728, 2006.

[48] C. Scherer, Robust mixed control and LPV control with full block scalings, ser. Advances in Design \& Control. Springer-Verlag, 1999, ch. 10, pp. 187-207.

[49] K.-C. Goh, M. Safonov, and G. Papavassilopoulos, "A global optimization approach for the BMI problem," in Proc. IEEE Conf. Decision \& Control, vol. 3, 1994, pp. 2009-2014.

[50] A. Hassibi, J. How, and S. Boyd, "A path-following method for solving BMI problems in control," in Proc. American Control Conf., vol. 2, 1999 , pp. $1385-1389$

[51] T. Iwasaki and R. Skelton, "The XY-centring algorithm for the dual LMI problem: a new approach to fixed-order control design," Int. J. Control, vol. 62, pp. 1257-1272, 1995.

[52] D. Nešić, A. Teel, and E. Sontag, "Formulas relating $\mathcal{K} \mathcal{L}$ stability estimates of discrete-time and sampled-data nonlinear systems," Syst. Control Lett., vol. 38, pp. 49-60, 1999.

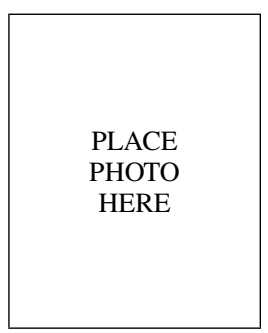

Tijs Donkers (SM '08) Tijs Donkers received the MSc degree (cum laude) in Systems and Controls in 2008 from Eindhoven University of Technology, the Netherlands.

Currently, he is with the department of Mechanical Engineering of the same university pursuing a $\mathrm{PhD}$ degree. His current research interests include networked and event-driven control, distributed control, and switched systems. 


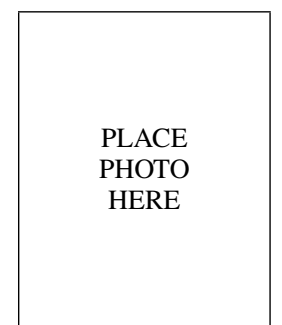

Maurice Heemels (M '06) received the MSc in mathematics (with honours) and the Ph.D. degrees (cum laude) from the Eindhoven University of Technology (TU/e), Eindhoven, the Netherlands, in 1995 and 1999, respectively.

From 2000 to 2004 , he was with the Electrical Engineering Department, TU/e, as an Assistant Professor and from 2004 to 2006 with the Embedded Systems Institute (ESI) as a Research Fellow. Since 2006, he has been with the Department of Mechanical Engineering,TU/e, where he is currently a Full Professor in the Hybrid and Networked Systems Group. He held visiting research positions at the Swiss Federal Institute of Technology (ETH), Zurich, Switzerland (2001) and at the University of California at Santa Barbara (2008). In 2004, he was also at the Research \& Development laboratory, Oc, Venlo, the Netherlands. His current research interests include hybrid and nonsmooth dynamical systems, networked control systems and constrained systems including model predictive control.

Dr. Heemels is an Associate Editor for the journals Automatica and Nonlinear Analysis: Hybrid Systems and serves in the international program committees of various conferences.

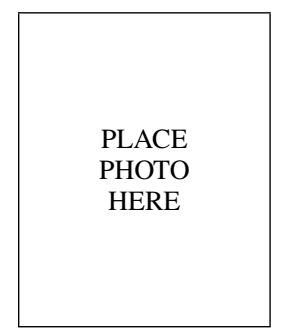

Nathan van de Wouw (M '08) received the MScdegree (with honours) and $\mathrm{PhD}$-degree in Mechanical Engineering from the Eindhoven University of Technology, Eindhoven, the Netherlands, in 1994 and 1999, respectively.

From 1999 until 2010, he has been affiliated with the Department of Mechanical Engineering of the Eindhoven University of Technology in the group of Dynamics and Control as an assistant/associate professor. In 2000, he has been working at Philips Applied Technologies, Eindhoven, the Netherlands, and, in 2001, he has been working at the Netherlands Organisation for Applied Scientific Research (TNO), Delft, The Netherlands. He has held positions as a visiting professor at the University of California Santa Barbara, USA, in 2006/2007 and at the University of Melbourne, Australia, in 2009/2010.

$\mathrm{He}$ has published a large number of journal and conference papers and the books 'Uniform Output Regulation of Nonlinear Systems: A convergent Dynamics Approach' with A.V. Pavlov and H. Nijmeijer (Birkhauser, 2005) and 'Stability and Convergence of Mechanical Systems with Unilateral Constraints' with R.I. Leine (Springer-Verlag, 2008). His current research interests are the analysis and control of nonlinear/non-smooth systems and networked control systems.

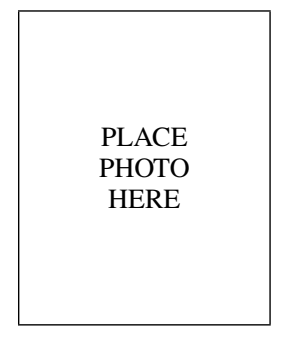

Laurentiu Hetel received the $\mathrm{PhD}$ Degree in $\mathrm{Au}$ tomatic Control from the Institut National Polytechnique de Lorraine, Nancy University, France, in 2007.

Since October 2008, he has been Associate Scientist (Chargé de Recherche) with the Centre National de la Recherche Scientifique (CNRS). He works at the Laboratoire d'Automatique, Génie Informatique et Signal (LAGIS) in Lille, France. His main research interests include the analysis and control of hybrid and embedded systems. 